This item was submitted to Loughborough's Research Repository by the author.

Items in Figshare are protected by copyright, with all rights reserved, unless otherwise indicated.

\title{
Phase-field modeling of eutectic structures on the nanoscale: the effect of anisotropy
}

PLEASE CITE THE PUBLISHED VERSION

https://doi.org/10.1007/s10853-017-0853-8

\section{PUBLISHER}

(C) Springer

\section{VERSION}

AM (Accepted Manuscript)

\section{PUBLISHER STATEMENT}

This work is made available according to the conditions of the Creative Commons Attribution-NonCommercialNoDerivatives 4.0 International (CC BY-NC-ND 4.0) licence. Full details of this licence are available at: https://creativecommons.org/licenses/by-nc-nd/4.0/

\section{LICENCE}

CC BY-NC-ND 4.0

\section{REPOSITORY RECORD}

Ratkai, Laszlo, Gyula Toth, Laszlo Kornyei, Tamas Pusztai, and Laszlo Granasy. 2019. "Phase-field Modeling of Eutectic Structures on the Nanoscale: The Effect of Anisotropy". figshare. https://hdl.handle.net/2134/27269. 


\section{Phase-field modeling of eutectic structures on the nanoscale: the effect of anisotropy}

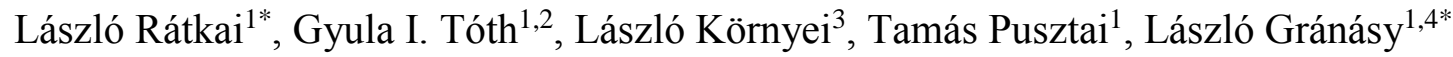

${ }^{1}$ Wigner Research Centre for Physics, Hungary

${ }^{2}$ University of Bergen, Norway

${ }^{3}$ Széchenyi University, Hungary

${ }^{4}$ Brunel University, United Kingdom

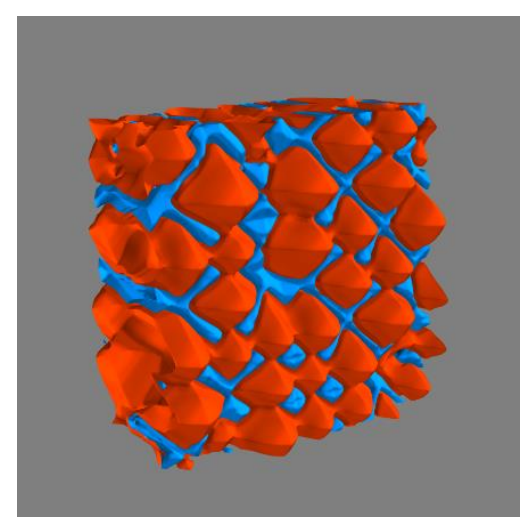

Eutectic solidification front formed in the presence of a highly anisotropic solid-liquid interfacial free energy. It has been demonstrated that the eutectic pattern is sensitive to the morphology of the solid-liquid interface. 


\section{Phase-field modeling of eutectic structures on the nanoscale: the effect of anisotropy}

László Rátkai ${ }^{1}(\bowtie)$, Gyula I. Tóth ${ }^{1,2}$, Tamás Pusztai ${ }^{1}$, László Környei ${ }^{3}$, and László Gránásy ${ }^{1,4}(\bowtie)$

1 Wigner Research Centre for Physics, P.O.Box 49, H-1525 Budapest, Hungary

2 Department of Physics and Technology, University of Bergen, Allégaten 55, 7005 Bergen, Norway

3 Department of Mathematics and Computational Sciences, Széchenyi István University,H-9026 Győr, Hungary

4 Brunel Centre for Advanced Solidification Technology, Brunel University, Uxbridge, Middlesex, UB8 3PH, UK

\section{ABSTRACT}

A simple phase-field model is used to address anisotropic eutectic freezing on the nanoscale in two (2D) and three dimensions (3D). Comparing parameter-free simulations with experiments, it is demonstrated that the employed model can be made quantitative for Ag-Cu. Next, we explore the effect of material properties, and the conditions of freezing on the eutectic pattern. We find that the anisotropies of kinetic coefficient and the interfacial free energies (solid-liquid and solid-solid), the crystal misorientation relative to pulling, the lateral temperature gradient, play essential roles in determining the eutectic pattern. Finally, we explore eutectic morphologies, which form when one of the solid phases are faceted, and investigate cases, in which the kinetic anisotropy for the two solid phases are drastically different.

\section{KEYWORDS:}

Ultrafine eutectics, nanoscale eutectics, anisotropy, pattern formation, phase-field theory, theory vs. experiment.

Address correspondence to László Rátkai, ratkai.laszlo@wigner.mta.hu; and László Gránásy, granasy.laszlo@wigner.mta.hu 


\section{Introduction}

Ultrafine eutectic structures (of sub-micron eutectic wavelength) have drawn considerable interest recently, owing to their outstanding mechanical [1-5], and unique optical properties [6-13] associated with the fine-scale (micron, submicron) distribution of the eutectic phases. This interest has been manifested in research projects such as the EU FP7 "ENSEMBLE" [11] aimed at creating eutectic metamaterials of special optical properties (e.g., negative refractive index, etc. [6-13]). A variety of eutectic patterns are regarded as promising candidates [6-17].

During the past decade, phase-field modelling contributed substantially to a better understanding of factors determining the eutectic patterns. Advances in this field are reviewed in Refs. [18-21]. Most of the previous studies considered isotropic solid-solid and solid-liquid interfaces. A broad variety of the eutectic systems show interfaces with anisotropic interfaces. Recent papers [22-26] address the effect of anisotropy of the solid-solid and solid-liquid interfaces on the eutectic pattern; reporting e.g., the existence of locked and unlocked relative orientations in the case of supercritical anisotropy [22-24].

Characteristic features of the eutectic meta-materials based on ceramic materials include fine/ultrafine patterns, high anisotropy, and often the presence of line compounds (compounds of strict stoichiometry) [27]. During the course of the ENSEMBLE project [13], we addressed the formation of various eutectic patterns using phase-field techniques (a few examples are shown in Fig. 1). Depending on the size scale of the problem, we used different formulations of the phase-field approach: the one whose results are presented here relies on two fields: a phase field, $\phi(\mathbf{r}, \mathrm{t})$, which monitors the solid-liquid transition, and a concentration field, $c(\mathbf{r}, \mathrm{t})$, characterizing the local composition. Square-gradient (SG) terms were included into the free energy density for both fields. This model was applied up to the micrometer and microsecond scales, while assuming physical interface thickness $(\sim 1 \mathrm{~nm})$, thus there was no need to correct for an artificially broad interface, often employed in quantitative phase-field modelling, when larger scale solidification is addressed [28-32]. 
Herein, we investigate the effects on various types of anisotropies on the eutectic pattern in the framework of the two-field model mentioned above relying on a physical interface thickness, which is therefore expected to be quantitative on nanoscale.

\section{Model applied}

The model used here is an extension of a phase-field model described in [33] (itself originating from [34]), obtained by adding a $\nabla^{2} c$ term to the free energy density and removing the free energy term associated with the orientation field. (For details of the derivation of the model see Refs. [33] and [34].)

$$
\begin{aligned}
F=\int d^{3} r\left\{\frac{\varepsilon_{\phi}^{2} T}{2} s_{\phi}^{2}\left(\vartheta_{\phi}, \theta_{\phi}\right)|\nabla \phi|^{2}+\frac{\varepsilon_{c}^{2} T}{2} s_{c}^{2}\left(\vartheta_{c}, \theta_{c}\right)|\nabla c|^{2}+w(c) T g(\phi)+[1-p(\phi)] f_{S}(c, T)+p(\phi) f_{L}(c, T)\right\} & \text { where } \\
\varepsilon_{\phi}^{2} & =\frac{6 \sqrt{2} \gamma_{A, B} \delta_{A, B}}{T_{A, B}}, \quad w(c)=(1-c) w_{A}+c w_{B}, \quad w_{A, B}=\frac{6 \sqrt{2} \gamma_{A, B}}{\delta_{A, B} T_{A, B}}, \\
g(\phi) & =\frac{1}{4} \phi^{2}(1-\phi)^{2}, \quad g^{\prime}(\phi)=\phi^{3}-\frac{3}{2} \phi^{2}+\frac{1}{2} \phi \\
p(\phi) & =\phi^{3}\left(10-15 \phi+6 \phi^{2}\right), \quad p^{\prime}(\phi)=30 \phi^{2}(1-\phi)^{2}, \\
s_{\phi, c, M} & \left(\vartheta_{\phi, c, M}, \theta_{\phi, c, M}\right)=1+\frac{s_{0, \phi, c, M}}{2} \cos \left(n \vartheta_{\phi, c, M}-2 \pi \theta_{\phi, c, M}\right), \\
\vartheta_{\phi, M} & =\arctan \left[(\nabla \phi)_{y} /(\nabla \phi)_{x}\right] \quad \operatorname{and} \quad \vartheta_{c}=\arctan \left[(\nabla c)_{y} /(\nabla c)_{x}\right], \\
f_{S . L} & =(1-c) f_{S, L}^{A}+c f_{S, L}^{B}+\frac{\left(\Omega_{S, L}^{0}+T \Omega_{S, L}^{1}\right) c(1-c)}{v_{m}}+\frac{R T}{v_{m}}\{c \log (c)+(1-c) \log (1-c)\} .
\end{aligned}
$$

Here $\gamma_{A, B}, \delta_{A, B}$, and $T_{A, B}$ denote the interface free energy and thickness of the equilibrium solid-liquid interface, and the melting point for the components A and B. T is the temperature, whereas $g(\phi)$ and $p(\phi)$ are the double well and interpolation functions, while ' stands for differentiation with respect to the argument of the function. $w$ and $w_{A, B}$ denote the free energy scales. $s_{\phi, c, M}$ and $s_{O \phi_{,}, M}$ are the anisotropy functions and strengths for the SG terms for the phase and concentration fields, and for the phase-field mobility $M_{\phi}$, whereas $\vartheta_{\phi, c, M}$ are the angle for the normal vector of the solid-liquid and solid-solid interfaces in the laboratory frame, while $\theta_{\phi, c, M}$ are the crystallographic orientation of the actual crystal grain, relative to which the respective anisotropy is measured. Unless stated otherwise the anisotropy functions shown above were used. Note that the present construction allows the assignment of different anisotropies to both SG terms and the phase-field mobility. While a very recent 
work [35] indicates that the handling of anisotropies can be far more complex owing to mechanical effects than the "usual" treatment adopted here, the latter proved to be a practical way to address anisotropy for various complex problems [36-39]. Anisotropy functions of the types employed here were used to represent the results from molecular dynamics studies [36, 40,41] and molecular scale theories [42-44]. Future work is planned to consider the influence of mechanical effects on the eutectic patterns.

In the majority of the present simulations, free energy densities $f_{S, L}$ taken from the regular solution model were adopted with temperature dependent interaction coefficients for the solid (S) and liquid (L) phases $\left(\Omega_{S, L}^{\emptyset}\right.$ and $\Omega$ ${ }_{S, L}$ ), which enables a reasonably accurate modelling of simple systems such as Ag-Cu. Here $R$ and $v_{m}$ are the gas constant and the molar volume. While $\varepsilon \phi^{2}$ is related to the properties of the solid-liquid interface, $\varepsilon_{c}^{2}$ controls the magnitude of free energy contribution from the spatial variation of the concentration field across the interfaces.

The corresponding equations of motion for the phase and concentration fields read as follows:

$$
\dot{\phi}=-M_{\phi} \frac{\delta F}{\delta \phi}=M_{\phi} \varepsilon_{\phi}^{2} T\left[\nabla\left(s_{\phi}^{2} \nabla \phi\right)-\frac{\partial}{\partial x}\left\{s_{\phi} \frac{\partial s_{\phi}}{\partial \vartheta_{\phi}} \frac{\partial \phi}{\partial y}\right\}+\frac{\partial}{\partial y}\left\{s_{\phi} \frac{\partial s_{\phi}}{\partial \vartheta_{\phi}} \frac{\partial \phi}{\partial x}\right\}-\frac{w(c) T g^{\prime}(\phi)+p^{\prime}(\phi)\left\{f_{L}(c, T)-f_{S}(c, T)\right\}}{\varepsilon_{\phi}^{2} T}\right]
$$

and

$$
\dot{c}=\nabla\left\{M_{c} \nabla \frac{\delta F}{\delta c}\right\}=\nabla\left\{\frac{v_{m}}{R T} D c(1-c) \nabla\left[\begin{array}{l}
\left(w_{B}-w_{A}\right) T g(\phi)+[1-p(\phi)] \frac{\partial f_{S}}{\partial c}(c, T)+p(\phi) \frac{\partial f_{L}}{\partial c}(c, T)- \\
-\varepsilon_{c}^{2} T\left(\nabla\left(s_{c}^{2} \nabla c\right)-\frac{\partial}{\partial x}\left\{s_{c} \frac{\partial s_{c}}{\partial \vartheta_{c}} \frac{\partial c}{\partial y}\right\}+\frac{\partial}{\partial y}\left\{s_{c} \frac{\partial s_{c}}{\partial \vartheta_{c}} \frac{\partial c}{\partial x}\right\}\right)
\end{array}\right]\right\}
$$

Here $\delta F / \delta X$ stands for the functional derivative of the free energy with respect to field $X(=\phi$ or $c), M_{\phi}=M_{\phi, 0}$ $s_{\mathrm{M}}\left(\vartheta_{M}, \theta_{M}\right)$ and $M_{c}=v_{m} D c(1-c) / R T$ are the phase field and concentration mobilities, whereas $D=D_{S}+\left(D_{L}-\right.$ $\left.D_{S}\right) p(\phi)$ is a phase-field-dependent diffusion coefficient.

With $c$-dependent switching functions $q(c)$ that vary between 0 and 1 monotonously, one may implement different anisotropies for the solid-liquid interfacial free energy or the phase-field mobility of the solid phases $\alpha$ 
and $\beta$ : e.g., $s_{\phi}=q(c) s_{\phi, \alpha}+[1-q(c)] s_{\phi, \beta}$. Eqs. 2 and 3 were solved numerically by two methods, with explicit finite difference (FD) method with Euler forward time stepping, and by a spectral method combined with operator splitting (SOS) [45]. Periodic boundary conditions were used in the latter case, in the case of FD various combinations of boundary conditions were applied. The computations were performed on a CPU cluster of 928 cores, and on several GPU cards of various types.

\section{Materials parameters}

We applied here two sets of thermodynamic properties for $\mathrm{Al}-\mathrm{Cu}$, one from the ThermoCalc database [46] that yields the phase diagram shown in Fig. 2a. These data were used in the simulations compared to the experimental data. The other set, is from the regular solution model leading to the phase diagram displayed in Fig. $2 \mathrm{~b}$. In the latter case, we have chosen the interaction parameters $\Omega_{S}{ }_{S}=26.0 \mathrm{~kJ} / \mathrm{mol}, \Omega^{\bigcap}=16.0 \mathrm{~kJ} / \mathrm{mol}, \Omega^{1}{ }_{S}=-4.0 \mathrm{~J} / \mathrm{mol} / \mathrm{K}$, and $\Omega^{1}{ }_{L}=-4.0 \mathrm{~J} / \mathrm{mol} / \mathrm{K}$, respectively, so that they yield a reasonable approximation of the phase diagram, $T_{E}=$ 1055.5 K and $c_{E}=0.35$ for the temperature and $\mathrm{Cu}$ concentration of the eutectic point. This set of data was used in mapping the solidification morphology.

Other materials parameters, we used were as follows. The solid/liquid interfacial free energies were taken from the compilation [47], $\gamma_{A g L}=172 \mathrm{mJm}^{-2}, \gamma_{C u L}=227 \mathrm{~mJ} \mathrm{~m}^{-2}$, which represent the average values deduced from dihedral angle measurements. The melting points used were taken from thermodynamic database [46]: $T_{A g}=1234$ $\mathrm{K}, T_{C u}=1358 \mathrm{~K}$, and the heat of fusions $\Delta H_{A g}=11.30 \mathrm{~kJ} \mathrm{~mol}^{-1}$, and $\Delta H_{C u}=13.05 \mathrm{~kJ} \mathrm{~mol}^{-1}$. A constant molar volume taken as the average of $v_{A g}=10.27 \mathrm{~cm}^{3} \mathrm{~mol}^{-1}$, and $v_{C u}=7.11 \mathrm{~cm}^{3} \mathrm{~mol}^{-1}$ was used [48]. In agreement with molecular dynamics simulations, the $10 \%-90 \%$ thickness of the interfaces were assumed to be $1 \mathrm{~nm}$ for both the solid-solid and solid-liquid interfaces. Herein, we assumed $\varepsilon_{c}^{2}=\varepsilon \phi^{2} / 4$. Finally, we adopted $D_{L}=1.4 \times 10^{-9}$ $\mathrm{m}^{2} \mathrm{~s}^{-1}$ for the liquid state and a 4 orders of magnitude smaller value for the solid phases $\left(D_{S}=D_{L} / 10^{4}\right)$. The phasefield mobility has been chosen as $M_{\phi}=0.25 \mathrm{~m}^{3}(\mathrm{Js})^{-1}$. Unless stated otherwise, isotropic interfacial properties were assumed. 


\section{Results and discussion}

\section{Comparison with experiment}

First, we investigate, whether the applied model can be regarded quantitative for a simple eutectic system such as $\mathrm{Ag}-\mathrm{Cu}$. With reasonable values of the materials parameters (from ThermoCalc), the velocity dependence of the eutectic wavelength $\lambda$ predicted by SOS implementation of the model is in a reasonable agreement with the experimental results [49] (see Fig. 3). Simulations with regular solution thermodynamics yield $\lambda^{2} v=0.4 \times 10^{-17}$ $\mathrm{m}^{3} \mathrm{~s}^{-1}$ for the Jackson-Hunt coefficient [50] (blue line), which is somewhat below the experimental value $\lambda^{2} v=$ $1.4 \times 10^{-17} \mathrm{~m}^{3} \mathrm{~s}^{-1}$ reported for Ag-Cu in the undercooling regime $\Delta T=19-104 \mathrm{~K}$ [49] (red line in Fig. 3). Apparently, with the present choice of model parameters a quantitative agreement is seen between theory and experiment.

Next, we investigated the morphologies the SOS version predicts in the highly undercooled state. Owing to the periodic boundary conditions used, we applied a temperature profile that has a constant section in the central region below $T_{E}$ and constant sections above $T_{E}$ that are connected with the central section by constant gradient sections. Thus the molten material traveling from above with a constant velocity first solidifies and then melts again. The results are summarized in Fig. 4. With increasing undercooling the following sequence of eutectic patterns was found: lamellae, lamellae mixed with globules, globules and merged horizontal globule pair, merged globules, and a "band-like" structure composed of lamellae parallel with the fronts. The latter essentially one dimensional eutectic growth mechanism is possible owing to the ease of the formation of the new phase due to the presence of the SG term for concentration in the free energy, allowing a spinodal-like transition. Lamellae close to perpendicular to growth were also seen for high cooling rate in splat cooling experiment [51]. 


\section{Anisotropy of $\varepsilon_{c}^{2}$ in two dimensions}

This part of our work has been motivated by Ref. 24. Here we used the regular solution approximant of Ag$\mathrm{Cu}$, however, the anisotropies were treated as adjustable parameters. First, we used the same anisotropy function as Ghosh et al., $s=1+s_{0} \cos \left[2\left(\vartheta-\theta_{0}\right)\right]$ [24], which was associated here exclusively with the SG term for the concentration field. No other quantities were made anisotropic, so in this section only, we omit the index $c$, which usually denotes that the anisotropy function refers to the $(\nabla c)^{2}$ term. It is worth noting that, as there is concentration gradient at the solid-liquid interface too, this anisotropy influences not only the solid-solid interface but also the solid- liquid interfaces. The thermodynamic data were taken from the regular solution approximant, whereas the simulations were performed on a rectangular $8192 \times 1024$ grid with periodic boundary condition on all sides. The shorter side was chosen to be equal to $8 \lambda_{J H}$, where $\lambda_{J H}$ is the (natural) Jackson-Hunt wavelength. (Accordingly, we had 128 pixels within $\lambda_{J H}$.) Solidification was started by a central slab parallel with the shorter side, containing 8 lamellae. No temperature gradient was applied. The initial front direction is conserved here owing to the restriction by the boundary condition. To mimic the local situation in the experiments [23], in which a circular disc was rotated so that the solidification front was in a fixed temperature gradient, here we varied the orientation of the long axis of the anisotropy relative to the direction of the long side of the simulation box. Here $\theta_{0}=0$ applies, when the long axis of the anisotropy is parallel with the long side of the simulation box. To mimic the experiment with rotating disc, $\theta_{0}$ was varied with time with a constant angular velocity. Simulations were performed with constant and time dependent $\theta_{0}$.

The results are summarized in Figs. 5 and 6. Fig. 5 displays the eutectic patterns observed for different strengths of the anisotropy in the range $0 \leq s_{o} \leq 0.5$. The pattern changes substantially. Two critical anisotropy values were observed: (i) The usual critical anisotropy $\left[s_{0}=1 /\left(2^{n}-1\right)\right.$ for $n$-fold symmetry] that corresponds the limit for the occurrence of excluded orientations in the equilibrium shape (see e.g., [52]), yielding here 1/3. Beyond this value, sharp changes in the phase-boundary can be observed at $\theta_{0}= \pm \pi / 2, \pm 3 \pi / 2$, etc. (ii) A more drastic change in the pattern was observed for $s_{o c 1}>0.4125$, in which regime, two-dimensional eutectic growth switches to one- 
dimensional (lamellae parallel with the front) at $\theta_{0}= \pm \pi / 2$. As reported in Refs. [22-24], the tilting angle of the phase boundary may considerably differ from the actual tilting angle. This is demonstrated for different anisotropy strengths in Fig. 6, where for comparison, results for ideal locking (realization of the actual tilting angle), and predictions of an analytical formulation based on the assumption of a symmetric-pattern (SP) described in Refs. $[22,24]$ are also displayed. For small anisotropies $\left(s_{o} \leq 0.1\right)$, the present simulations are in an excellent agreement with the analytical SP model, and thus with the results from the boundary integral method [24] and from quantitative phase-field modelling based on the multi-phase concept [24]. However, the difference between the results of the SP model and the present simulations increases with increasing anisotropy (for $s_{o}>0.15$ ). While for nearly critical and supercritical anisotropies (e.g., $s_{o}=0.4$ ), the present results approximate full locking reasonably for small actual angles, at odd multiples of $\pm \pi / 2$ they are about perpendicular to each other. This is clearly visible in Fig. 7 displaying the actual $\theta_{0}$ by green arrows, which follow closely the $\alpha-\beta$ interphase boundary, except for the vicinity of $\theta_{0}= \pm \pi / 2, \pm 3 \pi / 2$, etc. In fact, for $s_{o}>s_{o, c}=1 / 3$, the SP model predicts a discontinuity in the lamella orientation, and the appearance of a new solution. The increasing deviation between the present phasefield predictions and the analytical model is probably associated with the fact that, as opposed to the assumption of the SP approximation, here the solid-liquid interfacial free energy is anisotropic owing to the anisotropy of the coefficient of $(\nabla c)^{2}$. Indeed, while the interfaces observed at small anisotropy are fairly symmetric, as opposed with the ones seen at large anisotropy that are rather asymmetric ( $c f$. Figs. 8a and $8 b)$. Work is underway to clarify the origin of these differences.

The results obtained above indicate that under appropriate conditions, the present phase-field model is in a quantitative agreement with experiments and other solutions from a quantitative phase-field model, analytic solution based on the symmetric pattern approximation, and the boundary integral method.

\section{Multiple anisotropies in two dimensions}

We attempted to model exotic eutectic structures, where the solid-liquid interface of one of the interfaces is faceted. We have chosen the example [43] shown in Fig. 9a. To model this morphology, we used a concentration 
dependent switching functions. For the minority phase, we assumed a supercritical anisotropy of 4-fold symmetry $\left(s_{O \phi \beta}=0.4\right)$, and a large 2-fold kinetic anisotropy $\left(s_{O M \beta}=1.98\right)$, whereas the majority phase was kept isotropic $\left(s_{O \phi \alpha}=0.0\right.$ and $\left.s_{O M \alpha}=0.0\right)$. The respective solidification morphology is shown in Fig. $9 \mathrm{~b}$. This morphology is transient both in the experiment and the simulation as the initial lead of the faceted phase disappears with time, and the isotropic phase catches up with the needles as the liquid depletes in the component dominant in the faceted phase. This similarity indicates that reverse engineering in terms of anisotropy functions can model fairly complex situations.

\section{Anisotropy of $\varepsilon_{c}^{2}$ in three dimensions}

In three dimensions (3D), straightforward generalizations of Eqs. 2 and 3 were solved using the FD and SOS schemes. The model has been adopted for directional solidification: material flow with a uniform velocity and a temperature gradient both parallel to the growth direction were incorporated. On the lateral surfaces periodic boundary condition was applied, while liquid of given composition (which was made to vary between $x_{C u}=0.2$ and 0.8) of given temperature entered at the side ahead of the front. We assumed that the diffusion in the solid is orders of magnitude smaller than in the liquid, and is thus negligible on the time scale studied, so, unless stated otherwise, we employed $D_{S}=0$. We implemented an automatism, which kept the position of the solidification front essentially at a fixed position in the simulation box. The coefficient of the SG term for the concentration field was assumed to be orientation dependent. In 3D, we applied the following single-parameter anisotropy function:

$$
s_{c}=\left\{1-3 s_{0, c}+s_{0, c} \frac{4\left[c_{x}^{4}+c_{y}^{4}+c_{z}^{4}\right]}{\left(c_{x}^{2}+c_{y}^{2}+c_{z}^{2}\right)^{2}}\right\}
$$

Here the notation $\nabla c=\left\{c_{x}, c_{y}, c_{z}\right\}$ is used, whereas $s_{0}$ stands for the strength of the anisotropy.

Anisotropy of $(\nabla c)^{2}$ : The solidification morphologies obtained varying the magnitude and sign of the anisotropy $\varepsilon_{0}$ are shown in Fig. 10 for the same volume fractions of the solid phases. (These are indeed nanoscale 
eutectic structures. We note in this respect that a recent analysis [54] suggests that for e.g., Al-Cu the minimum attainable lamella spacing might be as low as $3.7 \mathrm{~nm}$.) Apparently, the anisotropies tilted forward by $22.5^{\circ}$ prefer horizontal lamellae for $s_{0, \mathrm{c}}<0$, whereas for $s_{0, \mathrm{c}}>0$ irregular quadrangles were observed. For positive and negative anisotropy strengths $\left(s_{0, \mathrm{c}}=-0.3\right.$ and 0.3$)$, we explored how the tilting angle (which was set to $0^{\circ}, 22.5^{\circ}$, and $45^{\circ}$ ) influences pattern formation (Fig. 11). In both cases, large tilting yielded lamellar structures, however that are perpendicular to each other. The effect of volume fraction under similar condition is shown for three values of $s_{0}$ $(-0.1,0$, and 0.1$)$ in Fig. 12. Depending on anisotropy, with increasing volume fractions we see different sequences of patterns: $s_{0, \mathrm{c}}=-0.1:$ broken lamellae $\rightarrow$ lamellae with line and point defects $\rightarrow$ thin lamellae that break up; for $s_{0, \mathrm{c}}=0:$ rods $\rightarrow$ disordered lamellae $\rightarrow$ rods of the other phase; and $s_{0, \mathrm{c}}=0.1:$ irregular quadrangles $\rightarrow$ disordered lamellae $\rightarrow$ lamellas with defects $\rightarrow$ rods aligned into lines. The line and point defects in these simulations occur as a result of a dynamic evolution of the eutectic patterns due to the compositional change of the incoming liquid.

\section{Tailoring the eutectic pattern}

One of the structures identified as desirable for some applications can be approximated by a microstructure alternating between the rod and lamellar structures. We attempted to produce such periodic rod-lamellar transitions along the pulling direction of the sample. A straightforward possibility (that is, however, not easy to realize experimentally) is to use an oscillatory composition for the liquid entering the simulation box. Choosing appropriate limits for this oscillation, periodical transition from the rod structure to the lamellar and backward can be achieved. We performed simulations for an isotropic system. Our results indicate that oscillatory temperature gradient may have a similar effect.

Unfortunately, the lamellar structure was fairly random as might have been expected in such a case (see Fig. 13a). One possibility to make it regular is to apply a cross-sectional temperature gradient [45]. We opted for a sinusoidal temperature change in the x direction (horizontal in Fig. 13b). Indeed, a fairly regular lamellar structure 
was obtained, however, the lamellae are yet connected, which - we believe - can be avoided by varying the average composition of the liquid entering the simulation box.

\section{Anisotropy of the solid-liquid interface in three dimensions}

The investigations in two dimensions indicated that the morphology of the solid-liquid interface may have an essential role in determining the eutectic pattern. To investigate this possibility, we assigned different anisotropies to the solid-liquid interfaces. An anisotropy function,

$$
s_{\phi}=\left\{1-3 s_{0, \phi}+s_{0, \phi} \frac{4\left[\phi_{x}^{4}+\phi_{y}^{4}+\phi_{z}^{4}\right]}{\left(\phi_{x}^{2}+\phi_{y}^{2}+\phi_{z}^{2}\right)^{2}}\right\},
$$

analogous to Eq. 4, has been applied in the coefficient of the $(\nabla \phi)^{2}$ term of the free energy density leading to anisotropic solid-liquid interfacial free energy. It has been confirmed that indeed the interface morphology and the evolving eutectic pattern are closely coupled. A few examples are shown in Fig. 14. However, with sufficiently high temperature gradient, the roughness of the solid-liquid interface can be suppressed (Fig. 15).

An interesting case, where the importance of interface anisotropy is uncertain is the formation of two-phase spiralling dendrites in ternary alloys [56]. Using a ternary version of the present model for a model system, in which ideal solution thermo-dynamics is used in the liquid and the regular solution model in the solid phase, we were able to capture this morphology and pattern, however, assuming kinetic anisotropy (Fig. 16) [57]. A later analytical treatment though suggests that no anisotropy is needed for the formation of the two-phase dendrites [58]. A subsequent phase-field study of ours investigated this issue in some details [26], and still supports the importance of anisotropy in the formation of steady-state ternary eutectic dendrites: Without anisotropy, the emerging cellular structure does not display regular steady state spiralling. 


\section{Summary}

The effect of anisotropies of the interfacial properties on eutectic pattern formation has been investigated. We demonstrated that (i) the applied simple phase-field model can be made quantitative for Ag-Cu; (ii) for small anisotropy of the solid-solid interface, the predictions are in a good agreement with those of a quantitative multiphase-field theory, the boundary integral method, and an analytical solution based on the symmetric-pattern approximation; (iii) with appropriate choice of anisotropies, experimental morphologies observed in a system consisting of a faceted minority and a non-faceted majority phase can be recovered; (iv) it has been demonstrated that the uneven morphology of the solid-liquid interface influences strongly the eutectic pattern; and (v) the anisotropies play an essential role in determining the eutectic patterns.

\section{Acknowledgments}

This work has been supported by the National Agency for Research, Development, and Innovation (NKFIH), Hungary under contract OTKA-K-115959, and by the EU FP7 EU FP7 projects "ENSEMBLE" (Grant Agreement NMP4-SL-2008-213669) and "EXOMET" (contract No. NMP-LA-2012-280421, co-funded by ESA).

\section{Conflicts of interest}

The authors declare that they have no competing financial interests. 


\section{References}

[1] He G, Eckert J, Löser W, Schultz L (2003) Novel Ti-base nanostructure-dendrite composite with enhanced plasticity. Nat Mater 2:33-37.

[2] Louzguine DV, Kato H, Inoue A (2004) High-strength hypereutectic Ti-Fe-Co bulk alloy with good ductility. Philos Mag Lett 84:359-364.

[3] Park JM, Kim DH, Kim KB, Kim WT (2007) Deformation-induced rotational eutectic colonies containing length-scale heterogeneity in an ultrafine eutectic $\mathrm{Fe}_{83} \mathrm{Ti}_{7} \mathrm{Zr}_{6} \mathrm{~B}_{4}$ alloy. Appl Phys Lett 91:131907-1-3.

[4] Park JM, Mattern N, Kühn U, Eckert J, Kim KB, Kim WT, Chattopadhyay K, Kim DH (2009) High-strength bulk Al-based bimodal ultrafine eutectic composite with enhanced plasticity. J Mater Res 24:2605-2609.

[5] Samal S, Biswas K (2013) Novel high-strength NiCuCoTiTa alloy with plasticity. J Nanopart Res 15:1783$1-11$.

[6] Pawlak DA, Kolodziejak K, Turczynski S, Kisielewski J, Rozniatowski K, Diduszko R, Kaczkan M, Malinowski M (2006) Self-organized, rodlike, micrometer-scale micro-structure of $\mathrm{Tb}_{3} \mathrm{Sc}_{2} \mathrm{Al}_{3} \mathrm{O}_{12}-\mathrm{TbScO}_{3}:$ Pr eutectic. Chem Mater 18:2450-2457.

[7] Pawlak DA, Kolodziejak K, Diduszko R, Rozniatowski K, Kaczkan M, Malinowski M, Kisielewski J, Lukasiewicz $\mathrm{T}$ (2007) The $\mathrm{PrAlO}_{3}-\mathrm{Pr}_{2} \mathrm{O}_{3}$ eutectic, its microstructure, instability, and luminescent properties. Chem Mater 19:2195-2202.

[8] Pawlak DA, Kolodziejak K, Rozniatowski K, Diduszko R, Kaczkan M, Malinowski M, Piersa M, Kisielewski J, Lukasiewicz T (2008) $\operatorname{PrAlO}_{3}-\mathrm{PrAl}_{11} \mathrm{O}_{18}$ eutectic: its microstructure and spectroscopic properties. Cryst Growth \& Des 8:1243-1249. 
[9] Pawlak DA, Turczynski S, Gajc M, Kolodziejak K, Diduszko R, Rozniatowski K, Smalc J, Vendik I (2010) Metamaterials: How far are we from making metamaterials by self-organization? The microstructure of highly anisotropic particles with an SRR-like geometry. Adv Funct Mater 20:1116-1124.

[10] Sadecka K, Gajc M, Orlinski K, Surma HB, Klos A, Joywik-Biala I, Sobczak K, Dluyewski P, Toudert J, Pawlak DA (2015) When eutectics meet plasmonics: Nanoplasmonic, volumetric, self-organized, silverbased eutectic. Adv Optical Mater 3:381-389.

[11] K. Kolodziejak K, Gajc M, Rozniatowski K, Diduszko R, Pawlak DA (2016) Synthesis and structural study of a self-organized MnTiO3-TiO2 eutectic. J. Alloys \& Comp. 659: 152-158.

[12] Merino RI, Acosta MF, Orera, VM (2012) New polaritonic materials in the THz range made of directionally solidified halide eutectics. J Eur Ceram Soc 34:2061-2069.

[13] Pawlak DA (2008-2012) EU FP7 Collaborative Project ENSEMBLE, CF-FP 213669, Grant Agreement, Annex I. http://cordis.europa.eu/result/ren/56818_en.html

[14] Oliete PB, Peña JI, Larrea A, Orera, VM, Lorca JL, Pastor JY, Martín A, Segurado J (2007) Ultra-highstrength nanofibrillar $\mathrm{Al}_{2} \mathrm{O}_{3}-\mathrm{YAG}-\mathrm{YSZ}$ eutectics. Adv Mater 19: 2313-2318.

[15] Orera, VM, Merino RI, Pardo JA, Larrea A, de la Fuente G, Contreras L, Peña JI (2000) Oxide eutectics: Role of interfaces in the material properties. Acta Phys Slovaca 50:549-557.

[16] Pastor Y, Martin A, Molina-Aldareguia JM, Llorca J, Oliete PB, Larrea A, Peña JI, Orera VM, Arenal R (2013) Superplastic deformation of directionally solidified nanofibrillar $\mathrm{Al}_{2} \mathrm{O}_{3}-\mathrm{Y}_{3} \mathrm{Al}_{5} \mathrm{O}_{12}-\mathrm{ZrO}_{2}$ eutectics. J Eur Ceram Soc 33:2579-2586.

[17] Fullman RL, Wood DJ (1954) Origin of spiral eutectic structures. Acta Metall. 2:188-193.

[18] Steinbach I (2009) Phase-field models in materials science. Modelling Simul Mater Sci Eng 17:073001-131. 
[19] Nestler B, Choudhury A (2011) Phase-field modeling of multi-component systems. Current Opinion in Solid State and Mater Sci 15:93-105.

[20] Akamatsu S, Plapp M (2016) Eutectic and peritectic solidification patterns, Current Opinion in Solid State and Mater Sci 20:46-54.

[21] Boettinger WJ (2016) The solidification of multicomponent alloys. J Phase Equilibria and Diffusion 37:418.

[22] Akamatsu S, Bottin-Rousseau S, Serefoglu M, Faivre G (2012) A theory of thin lamellar eutectic growth with anisotropic interphase boundaries. Acta Mater 60:3199-3205.

[23] Akamatsu S, Bottin-Rousseau S, Serefoglu M, Faivre G (2012) Lamellar eutectic growth with anisotropic interphase boundaries Experimental study using the rotating directional solidification method. Acta Mater 60:3206-3214.

[24] Ghosh S, Choudhury A, Plapp M, Bottin-Rousseau S, Faivre G, Akamatsu S (2015) Interphase anisotropy effects on lamellar eutectics: a numerical study. Phys Rev E 91:022407-1-13.

[25] Lahiri A, Choudhury A (2015) Effect of surface energy anisotropy on the stability of growth fronts in multiphase alloys. Trans Indian Inst Met 68:1053-1057.

[26] Rátkai L, Szállás A, Pusztai T, Mohri T, Gránásy L (2015) Ternary eutectic dendrites: Pattern formation and scaling properties. J Chem Phys 142:154501-1-11.

[27] Llorca J, Orera V (2006) Directionally solidified eutectic ceramic oxides. Progr Mater Sci 51:711-809.

[28] Karma A (2001) Phase-field formulation for quantitative modeling of alloy solidification. Phys Rev Lett $87: 115701-1-4$.

[29] Echebarria B, Folch R, Karma A, Plapp M (2004) Quantitative phase-field model of alloy solidification. Phys Rev E 73:061604-1-22. 
[30] Hötzer J, Jainta M, Steinmetz P, Nestler B, Dennstedt A, Genau A, Bauer M, Köstler H, Rüde U (2015) Large scale phase-field simulations of directional ternary eutectic solidification. Acta Mater 93:194-204.

[31] Folch R, Plapp M (2005) Quantitative phase-field modeling of two-phase growth. Phys Rev E 72:011602$1-27$.

[32] Kim SG (2007) A phase-field model with antitrapping current for multicomponent alloys with arbitrary thermodynamic properties. Acta Mater 55:4391-4399.

[33] Gránásy L, Börzsönyi T, Pusztai T (2002) Nucleation and bulk crystallization in binary phase field theory. Phys Rev Lett 88:206105-1-4.

[34] Warren JA, Boettinger WJ (1995) Prediction of dendritc growth and microsegregation patterns in a binary alloy using the phase-field method. Acta Metall Mater 43:689-703.

[35] Levitas VI, Warren JA (2016) Phase field approach with anisotropic interface energy and interface stresses: Large strain formulation. J Mech Phys Solids 91:94-125.

[36] Hoyt JJ, Asta M, Karma A (2003) Atomistic and continuum modeling of dendritic solidification. Mater Sci Eng R 41: 121-163.

[37] Gránásy L, Pusztai T, Warren JA, Douglas JF, Börzsönyi T, Ferreiro V (2003) Growth of 'dizzy dendrites' in a random field of foreign particles. Nat Mater 2: 92-96.

[38] Gránásy L, Pusztai T, Börzsönyi T, Warren JA, Douglas JF (2004) A general mechanism of polycrystalline growth. Nat Mater 3: 645-650.

[39] Haxhimali T, Karma A, Gonzales F, Rappaz M (2006) Orientation selection in dendritic evolution. Nat Mater 5: 660-664.

[40] Davidchack R, Laird BB (2005) Direct calculation of the crystal-melt interfacial free energy via molecular dynamics computer simulation. J Phys Chem B 109:17802-17812. 
[41] Hoyt JJ, Asta M, Karma A (2001) Method for computing the anisotropy of the solid-liquid interfacial free energy. Phys Rev Lett 86:5530-5533.

[42] Majaniemi S, Provatas N (2009) Deriving surface-energy anisotropy for phenomenological phase-field models of solidification. Phys Rev E 79:011607-1-12.

[43] Hartel A, Oettel M, Rozas RE, Egelhaff SU, Horbach J, Löwen H (2012) Tension and stiffness of the hard sphere crystal-fluid interface. Phys Rev Lett 108:226101-1-4..

[44] Podmaniczky F, Tóth GI, Pusztai T, Gránásy L (2014) Free energy of the bcc-liquid interface and the Wulff shape as predicted by the phase-field crystal model. J Cryst Growth 385:148-153.

[45] Tegze G, Bansel G, Tóth GI, Pusztai T, Fan Z, Gránásy L (2009) Advanced operator-splitting-based semiimplicit spectral method to solve the binary phase-field crystal equation with variable coefficients. J Comput Phys 228:1612-1623.

[46] Andersson JO, Helander T, Höglund L, Shi PF, Sundman B (2002) Thermo-Calc and DICTRA, Computational tools for materials science. Calphad 26:273-312.

Thermo-Calc Software, http://www.thermocalc.com/

[47] Gránásy L, Tegze M (1991) Crystal-melt interfacial free energy of elements and alloys. Mater Sci Forum $77: 243-256$.

[48] Singman CN (1984) Atomic volume and allotropy of the elements. J Chem Edu 61:137-142.

[49] Cline HE, Lee D (1970) Strengthening of lamellar vs. equiaxed Ag-Cu eutectic. Acta Metall 18:315-323.

[50] Jackson KA, Hunt JD (1966) Lamellar and rod eutectic growth. Trans Metall Soc AIME 236:1129-1142.

[51] Galenko PK, Herlach DM (2006) Diffusionless crystal growth in rapidly solidifying eutectic systems. Phys Rev Lett 96:150602-1-4. 
[52] Kobayashi R, Giga Y (2001) On anisotropy and curvature effects for growing crystals. Jpn J Ind Appl Math 18:207-230.

[53] Jackson KA, Hunt JD Eutectic solidification in transparent materials, in Jackson KA Crystal Growth (video). Jackson KA (2011) Personal communication.

[54] Wang N, Trivedi R (2011) Limit of steady-state lamellar eutectic growth. Scripta Mater 64:848-851.

[55] Perrut M, Parisi A, Akamatsu S, Botin-Rousseau S, Faivre G, Plapp M (2010) Role of transverse temperature gradients in the generation of lamellar eutectic solidification patterns. Acta Mater 58:17611769.

[56] Akamatsu S, Perrut M, Bottin-Rousseau S, Faivre G (2010) Spiral two-phase dendrites. Phys Rev Lett 104:056101-1-4.

[57] Pusztai T, Rátkai L, Szállás A, Gránásy L (2013) Spiraling eutectic dendrites. Phys Rev E 87:032401-1-4

[58] Akamatsu S, Perrut M, Bottin-Rousseau S, Brener EA (2014) Scaling theory of two-phase dendritic growth in undercooled ternary melts. Phys Rev Lett 112:105502-1-4 


\section{Figure captions:}

Fig. 1 Eutectic patterns in directional solidification. Selection of pairs of micron scale experimental images (on the left) and snapshots of phase-field simulations (on the right) that reproduce similar patterns on the nanoscale (obtained within the EU FP7 project ENSEMBLE [13]) are shown. Experimental panels in the first column show: rods [6]; lamellae with line defects [15]; triangular structure [16]; fishnet structure [11]. In the third column the following structures are displayed: lamellae with point defects [7]; broken lamellae [7]; random rectangular splitring-resonator structure (horizontal size: $\sim 110 \mu \mathrm{m})$ [9]; spiraling eutectic growth $(\sim 20 \mu \mathrm{m} \times 15 \mu \mathrm{m})$ [17]. In the phase-field simulations, we varied the anisotropies of the $\alpha-\beta$ solid-solid, and $\alpha$ - liquid and $\beta$-liquid interfaces together with the composition, tilting angle, and pulling velocity. Although on much smaller scale, we were able to capture similar rod, and lamellar structure with point and line defects, the broken lamellae, and triangular motifs, and achieved some resemblance in the cases of the multi-scale split-ring resonators of rectangular C-like cross-section, the fish-net, and spiraling structures. Further work is needed in the case of the latter three to improve the similarity. The size of the simulation box used were as follows: In the second column: $40 \mathrm{~nm} \times 40 \mathrm{~nm} \times 20$ $\mathrm{nm}, 40 \mathrm{~nm} \times 40 \mathrm{~nm} \times 20 \mathrm{~nm}, 20 \mathrm{~nm} \times 20 \mathrm{~nm} \times 20 \mathrm{~nm}$, and $128 \mathrm{~nm} \times 128 \mathrm{~nm}$, respectively; while in the fourth column: $40 \mathrm{~nm} \times 40 \mathrm{~nm} \times 20 \mathrm{~nm}, 20 \mathrm{~nm} \times 20 \mathrm{~nm} \times 20 \mathrm{~nm}, 40 \mathrm{~nm} \times 40 \mathrm{~nm} \times 40 \mathrm{~nm}$, whereas for the lowest panel assuming a physical interface thickness in the dimensionless computation of Ref. 23, one obtains $95 \mathrm{~nm} \times 95 \mathrm{~nm}$ $\times 128 \mathrm{~nm}$ ). We note that eutectic structures such as rods and lamellae were seen to appear in a broad size range that covers several orders of magnitude when varying the pulling velocity, and following the Jackson-Hunt scaling, $\lambda^{2} v=$ const., where $\lambda$ is the eutectic wavelength, and $v$ the front velocity. Probably, similar relationship stands for the other eutectic patterns. 
Fig. 2 Ag-Cu phase diagrams: a. Computed using thermodynamic data from the ThermoCalc database [36], and b. from the regular solution model with parameters specified in the text.

Fig. 3 Eutectic wavelength vs velocity in the Ag-Cu system: Comparison of experiments (full symbols and solid line) [49] with phase-field (SOS version) predictions relying on ThermoCalc data (empty circles \& dashed line) and using the regular solution model $\left(\lambda^{2} v=0.4 \times 10^{-17} \mathrm{~m}^{3} \mathrm{~s}^{-1}\right.$; dotted line $)$. Apparently, the Jackson-Hunt scaling $\left(\lambda^{2} v=\right.$ const. [50], dashed line) is fairly closely followed.

Fig. 4 Eutectic patterns predicted by the present phase-field mode (SOS version) at extreme undercoolings at the front. The concentration field is shown, accordingly the color bar indicates changes between $c=0$ (black) and 1 (white). In these simulations the matter moves downwards, while solidification takes place at the upper front and melting occurs at the lower front. This was achieved by applying the following schematic temperature profile in the vertical direction: ${ }^{\neg}{ }^{-}$, where the highest and lowest temperatures were $1100 \mathrm{~K}$ and $T_{\mathrm{E}}-\Delta T$, respectively. At the solidification and melting fronts the temperature varied linearly with position. Here $D_{S}=0.01 D_{L}$ was used. Note the transition from lamellae to globules, and finally to a band-like pattern (lamellae parallel with the front). (The lamella spacing is $\sim 18 \mathrm{~nm}$ for the leftmost panel.) The size of the simulation box was $160 \mathrm{~nm} \times 320 \mathrm{~nm}$.

Fig. 5 Eutectic patterns predicted for rotating anisotropy. The concentration field is shown (the colourbar covers $c \in[0,1]$, the $\mathrm{Cu}$ reach phase is white; while the initial liquid concentration was $c=0.45$, and $\Delta T=150 \mathrm{~K})$. The numbers in the upper left corner indicate the strength of the anisotropy, $s_{o}$. Above a critical anisotropy $\left(s_{o}>s_{o, c}=\right.$ $1 / 3$ ), the orientation of the lamella varies sharply at $\theta_{0, c}= \pm \pi / 2, \pm 3 \pi / 2, \ldots$, while above another critical value ( $s_{o}$ $>s_{o, c l} \approx 0.4125$ ), one-dimensional eutectic growth is seen at $\theta_{0, c}= \pm \pi / 2$. (SOS scheme.) $8192 \times 1024$ grid was used. 
Fig. 6 Lamella angle vs. actual angle as a function of anisotropy. Besides the simulation results with constant rotation rate (empty symbols), and at fix tilting angles (full magenta symbols), the line corresponding to ideal locking (black dashed line), and results from analytical SP theory (colored solid lines) are also shown. Colors indicate the anisotropy strength.

Fig. 7 Eutectic pattern (concentration field, here yellow stands for the $\mathrm{Cu}$ reach phase) predicted by SOS version of the present phase-field model obtained with rotating anisotropy function for $s_{0}=0.4$ (the right half of the $6^{\text {th }}$ panel of Fig. 5 is shown). The green arrows show the orientation of the anisotropy function of 2-fold symmetry at the instance when the solidification front was there. Initially $(t=0), \theta_{0}=0$, i.e., the long axis of the anisotropy function is parallel with the initial phase boundaries. The long axis of anisotropy (denoted by the arrows) rotates counter-clockwise. At about $x=5500$ ( $x$ is the position in number of pixels from the left edge of the simulation box), the direction of the long axis of the anisotropy function becomes $\theta_{0}=\pi / 2$. Note that owing to the twofold symmetry of the anisotropy function antiparallel arrows indicate equivalent situations. (The right half of a simulation performed on an $8192 \times 1024$ grid is shown.)

Fig. 8. Growth fronts observed at small and large anisotropies (concentration map is shown; the yellow color stands for the $\mathrm{Cu}$-rich and the blue for the Ag-rich phases). a. $s_{0}=0.05$ (Fig. 5, $2^{\text {nd }}$ panel), and b. $s_{0}=0.4$ (Fig. 5 , $6^{\text {th }}$ panel). $200 \times 1024$ section of $8192 \times 1024$ simulations are shown.

Fig. 9 Eutectic morphology: small amount of faceted phase with majority non-faceted phase. a. Snapshot from the video [53]. (Size of the image is about $80 \mu \mathrm{m} \times 60 \mu \mathrm{m}$.) b. Phase-field simulation on a $4096 \times 1024$ grid with an isotropic black and a highly anisotropic white phase [characterized by a 2-fold kinetic anisotropy with $s_{0, M \beta}=$ 
1.98, and a 4-fold anisotropy for $(\nabla \phi)^{2}$ with $s_{0, \phi} \beta=-0.4$, tilting angles: $\theta_{0, \phi, M}=22.5^{\circ}$. (The SOS scheme was used; $c_{0}=0.3, \Delta T=200 \mathrm{~K}$, while $D_{S} / D_{L}=0.001$.)

Fig. 10 The effect of the anisotropy (upper row: large objects) of the phase boundary energy on the equilibrium (Wulff) shape (upper row: small objects to the left) and on the eutectic solidification pattern (lower row). FD scheme was used. From left to right $s_{0, c}=-0.3,-0.1,0.0,0.1$, and 0.3 , respectively. Coordinate axes: $x$ is parallel to growth direction; $y$ horizontal; and $z$ vertical. The phase-field simulations have been performed on a $160 \times 160$ $\times 80$ grid $(40 \mathrm{~nm} \times 40 \mathrm{~nm} \times 20 \mathrm{~nm})$, for $\mathrm{Ag}-\mathrm{Cu}$ at spatially uniform undercooling $\Delta T=155.5 \mathrm{~K}$, and assuming a diffusion coefficient of $D_{L}=1.4 \times 10^{-9} \mathrm{~m}^{2} \mathrm{~s}^{-1}$. The vertical axis of the anisotropy function was tilted forward by $22.5^{\circ}$. The yellow color indicates is the copper rich phase. Note the transition from faceted lamellae to regular rods, and then to faceted irregular quadrangles.

Fig. 11 The effect of tilting angle between the surface normal and the axis of anisotropy as a function of the anisotropy parameter (upper row: $s_{0, c}=-0.3$; lower row: $s_{0, c}=0.3$ ). From left to right, the tilting angle is $0^{\circ}, 22.5^{\circ}$, and $45^{\circ}$ along the horizontal axis. Other conditions are the same as for Fig. 10. Panels on the right show the respective Wulff shapes.

Fig. 12 The effect of the varying volume fraction on the eutectic pattern formation as a function of the anisotropy parameter. Upper row: $s_{0, c}=-0.1$; central row: $s_{0, c}=0$; lower row: $s_{0, c}=0.1$. The volume fraction of the light colored phase increases from left to right $(c \approx 0.30,0.5,0.7$, and 0.75 ; in the rightmost column deviation from the nominal volume fraction along the interface is due to its wavy shape). Other conditions are the same as for Fig. 10. Note the transition from horizontal to vertical lamellae via disordered ones as $s_{0, c}$ increases in the $4^{\text {th }}$ column. With further increasing of the volume fraction, these patterns break up into ordered or disordered chains of rods. 
Fig. 13 Three dimensional structure of one of the solid phases formed in directional solidification using periodically changing composition for the liquid entering the simulation performed (FD discretizaton was used): (a) Without and (b) with cross-sectional temperature gradient. Note the regularization of the lamellar structure. Another effect of cross-sectional temperature gradient is the declination of the rods from the pulling direction. Here $s_{0, c}=0$. The size of the simulation structures are given in $\mathrm{nm}$. The thickness of the simulation window was $20 \mathrm{~nm}$. The solid structure that left the simulation box at its back surface of were used to create these structures. Other conditions were the same as for Fig. 10.

Fig. 14 The effect of uneven surface on the eutectic pattern in the phase-field model (FD discretization): 1st row: composition and morphology of the solidification front (the $\phi=0.5$ surface is shown). Coordinate axes: $x$ is parallel to growth direction; $y$ horizontal; and $z$ vertical. $2^{\text {nd }}$ row: the cross-sectional $(y-z)$ distribution of composition (yellow and blue correspond to the eutectic phases). The size of the simulation box was $32 \mathrm{~nm} \times 32$ $\mathrm{nm} \times 32 \mathrm{~nm}$. Conditions for the three columns: Left: $c=0.775, s_{0, \phi}=s_{0, c}=0.3$, with a $45^{\circ}$ rotation of the anisotropy function for the $(\nabla c)^{2}$ term around the $x$-axis. Centre: $c=0.45, s_{0, \phi}=s_{0, c}=0.3$, with a $45^{\circ}$ rotation of the anisotropy function for the $(\nabla \phi)^{2}$ term around the $x$-axis. Right: $c=0.775, s_{0, \phi}=-0.3$ with a $6^{\circ}$ rotation of the anisotropy function for the $(\nabla \phi)^{2}$ term around the $y$-axis, and $s_{0, c}=0.3$ with a $45^{\circ}$ rotation of the anisotropy function for the $(\nabla c)^{2}$ term around the $x$-axis. Note the two size scales that correlate with the ledges observed in the latter case.

Fig. 15 Snapshots showing quantitative $3 D$ eutectic simulations with anisotropic solid-liquid interfacial free energy $\left(s_{0, \phi}=0.1\right)$ performed in the presence of different thermal gradients parallel with pulling. The concentration field is shown (blue and red corresponds to the eutectic compositions). [The computations were performed on a rectangular grid of $64 \mathrm{~nm} \times 64 \mathrm{~nm} \times 64 \mathrm{~nm}$ size, using advanced numerical methods (SOS scheme run on GPU 
cards), using thermodynamic data of $\mathrm{Ag}-\mathrm{Cu}$ taken from ThermoCalc [36], and a physical interface thickness, whereas the undercooling was $\Delta T=155 \mathrm{~K}$, while a pulling velocity $v=0.01 \mathrm{~m} \mathrm{~s}^{-1}$ was applied. The temperature gradient increases from left to right.]

Fig. 16 Tip of spiraling eutectic dendrites. From left to right the free energy of the solid-liquid interface, and thus the tip radius increases. Composition maps are shown (blue and red denote the eutectic phases). The front view ( $1^{\text {st }}$ row), the lateral section $\left(2^{\text {nd }}\right.$ row $)$, the side view $\left(3^{\text {rd }}\right.$ row $)$, and the cross-sectional composition distribution ( $4^{\text {th }}$ row) are shown. (FD scheme.) The computations were performed on a $96 \times 96 \times 612$ rectangular grid, assuming a physical interface thickness, this corresponds to about $24 \mathrm{~nm} \times 24 \mathrm{~nm} \times 153 \mathrm{~nm}$. For other details of the simulations see Ref. [57]. 


\section{Figures:}

Fig. 1
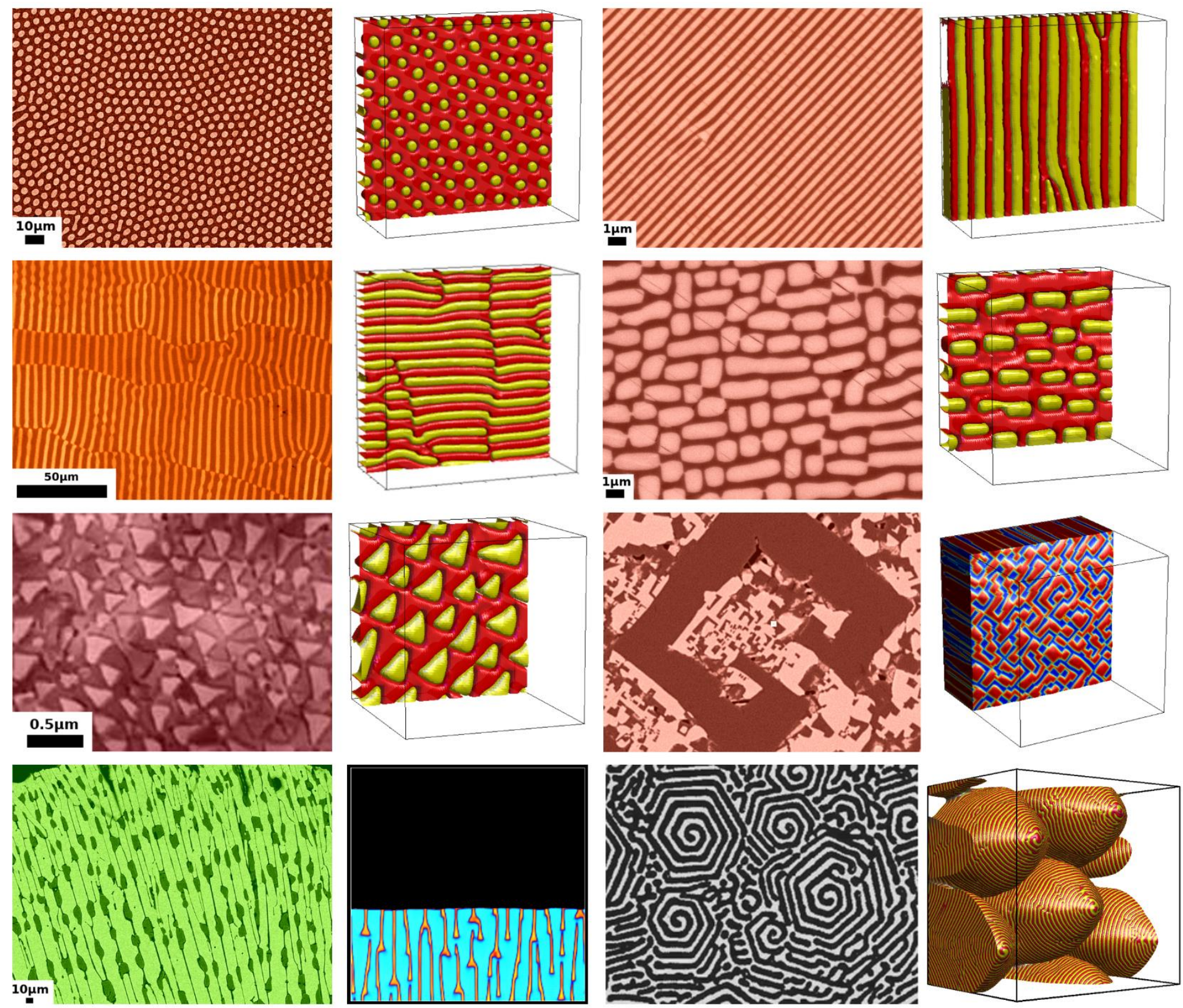
Fig. 2
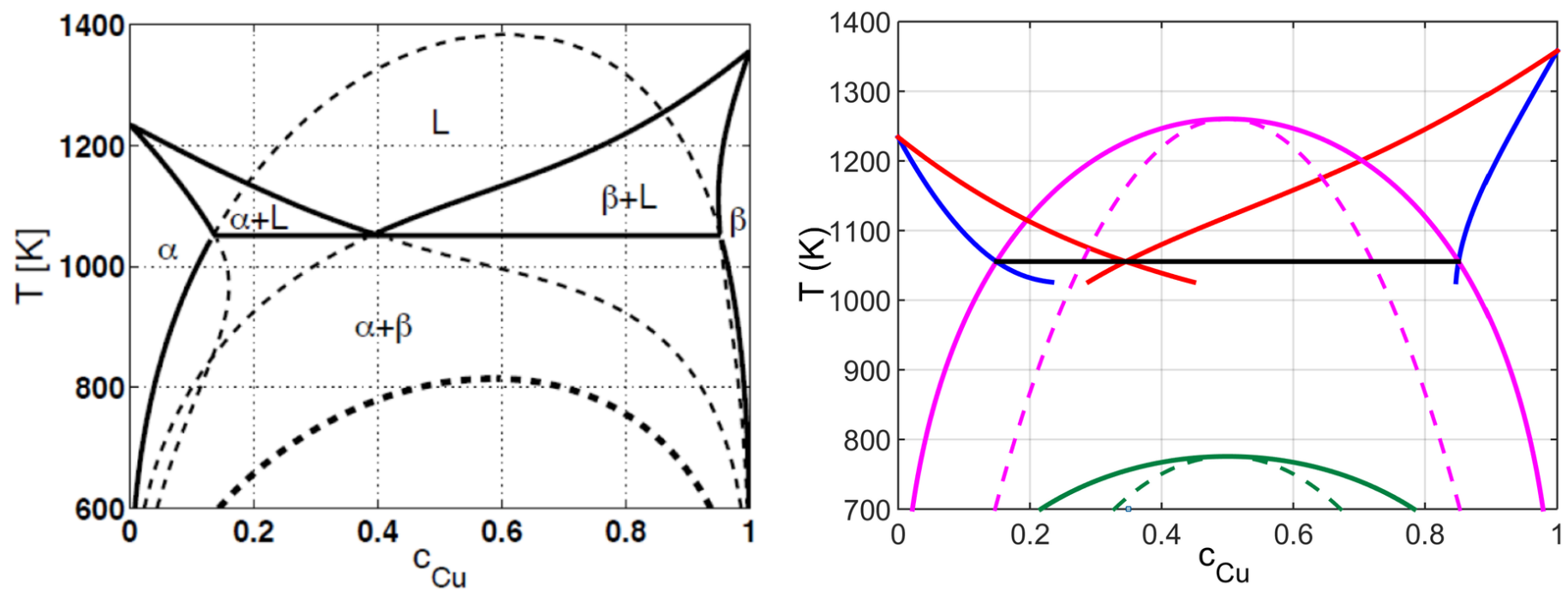
Fig. 3

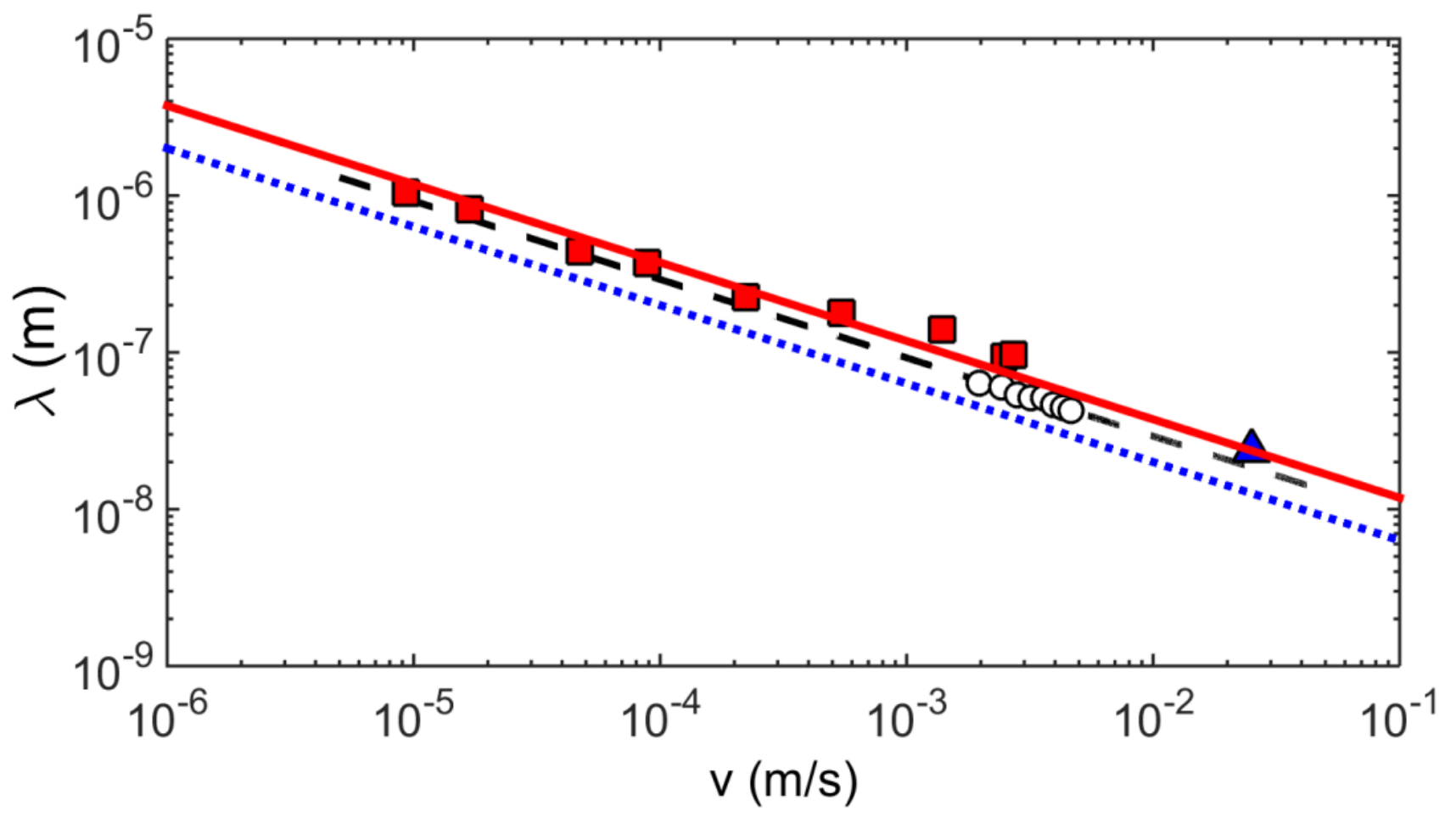


Fig. 4
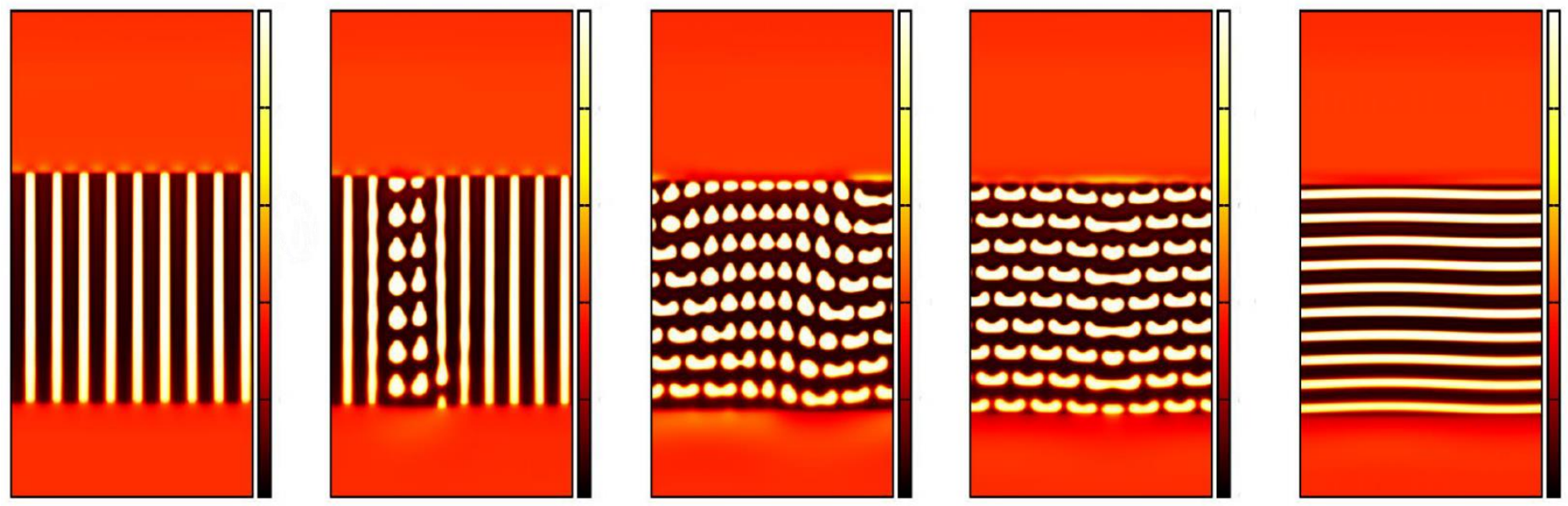

$$
\begin{array}{lll}
\Delta T=270[\mathrm{~K}], & \Delta T=280[\mathrm{~K}], & \Delta T=290[\mathrm{~K}], \\
v_{\text {pull }}=4.5[\mathrm{~cm} / \mathrm{s}] & v_{\text {pull }}=5.0[\mathrm{~cm} / \mathrm{s}] & v_{\text {pull }}=5.5[\mathrm{~cm} / \mathrm{s}]
\end{array}
$$$$
\Delta T=300[K],
$$$$
\Delta T=310[K],
$$$$
v_{\text {pull }}=6.0[\mathrm{~cm} / \mathrm{s}]
$$$$
v_{\text {pull }}=6.5[\mathrm{~cm} / \mathrm{s}]
$$ 
Fig. 5
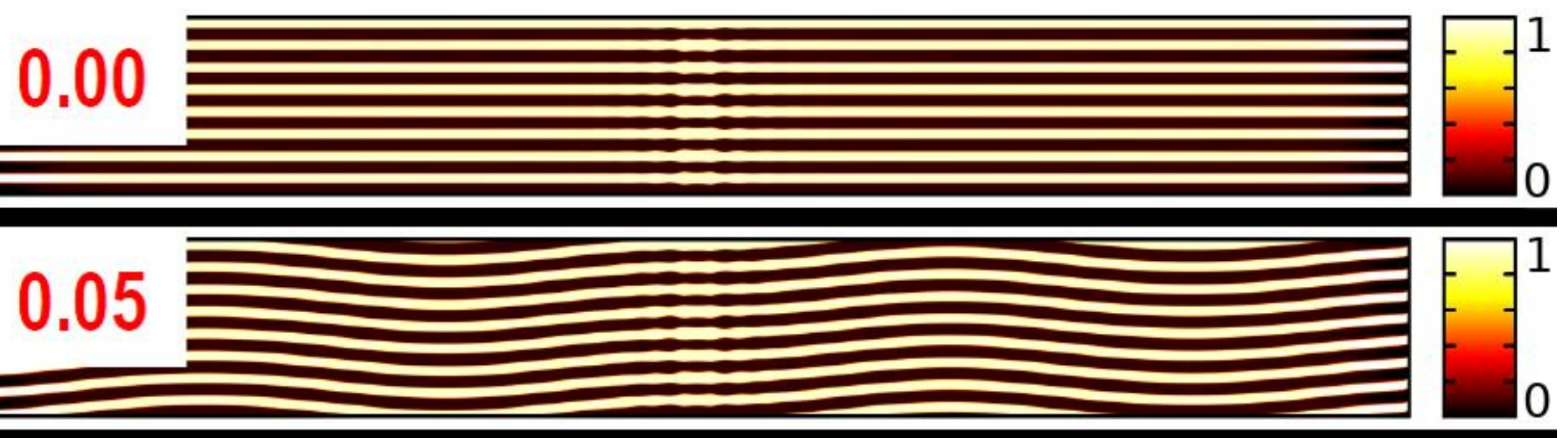

-
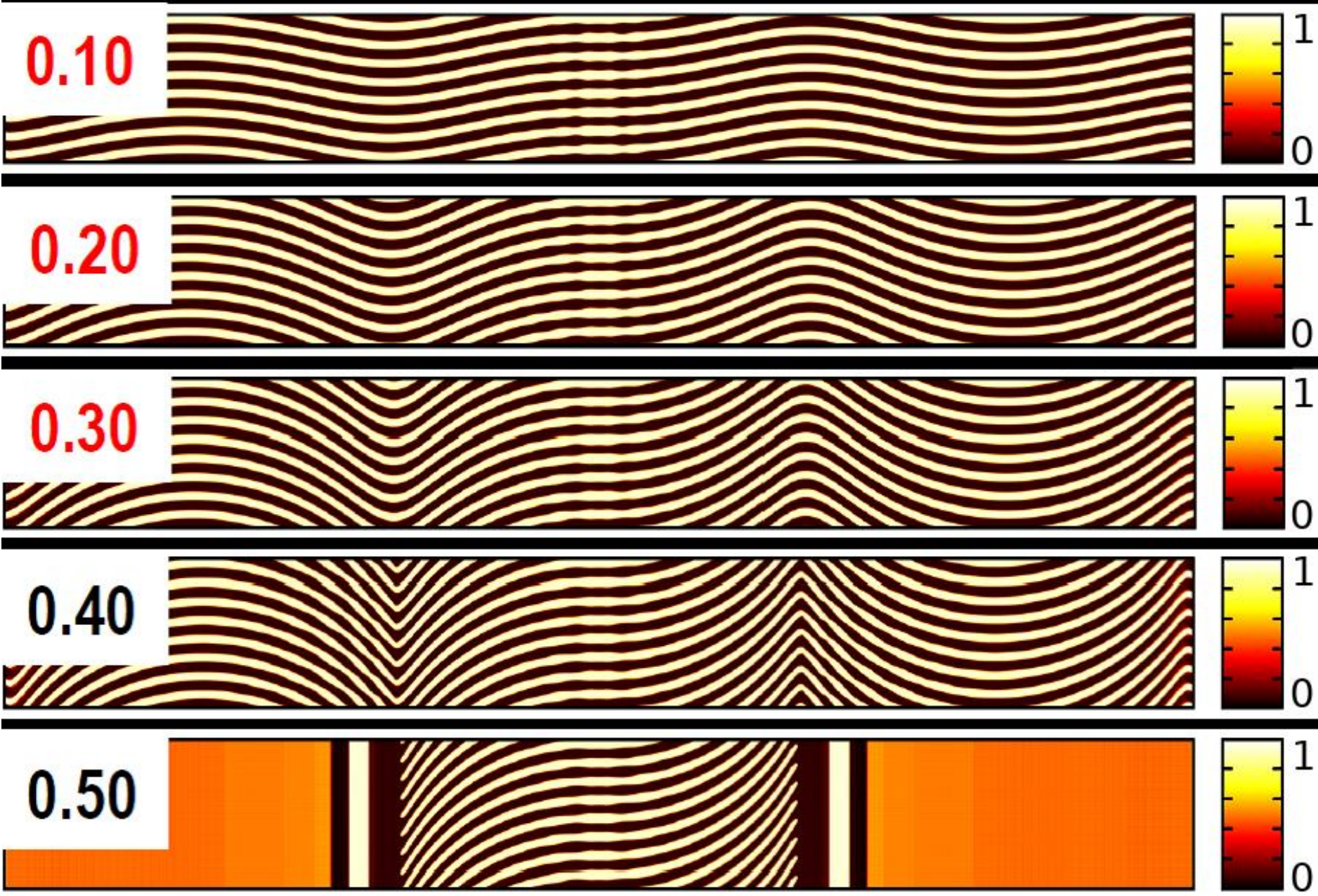
Fig. 6

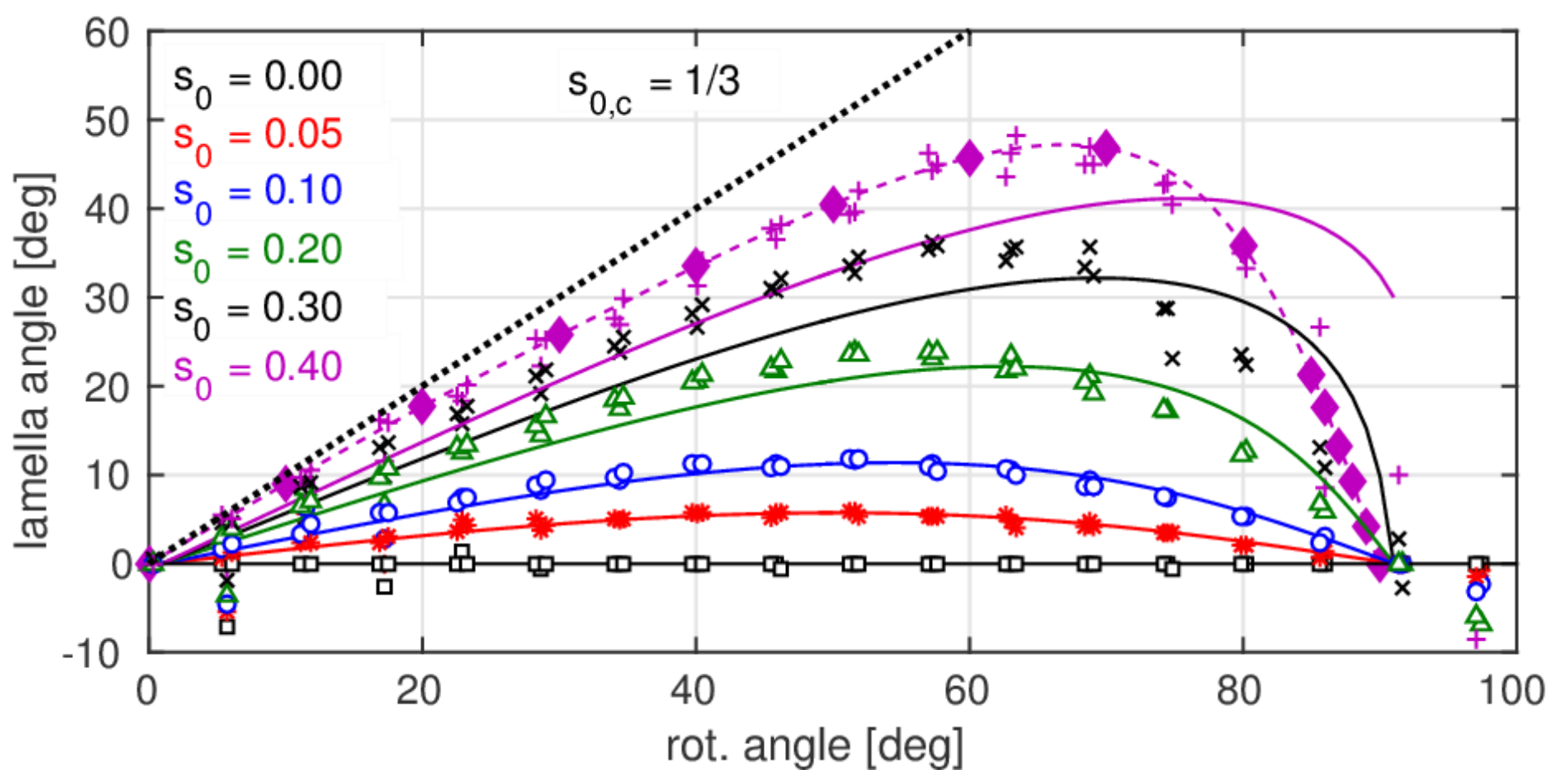


Fig. 7

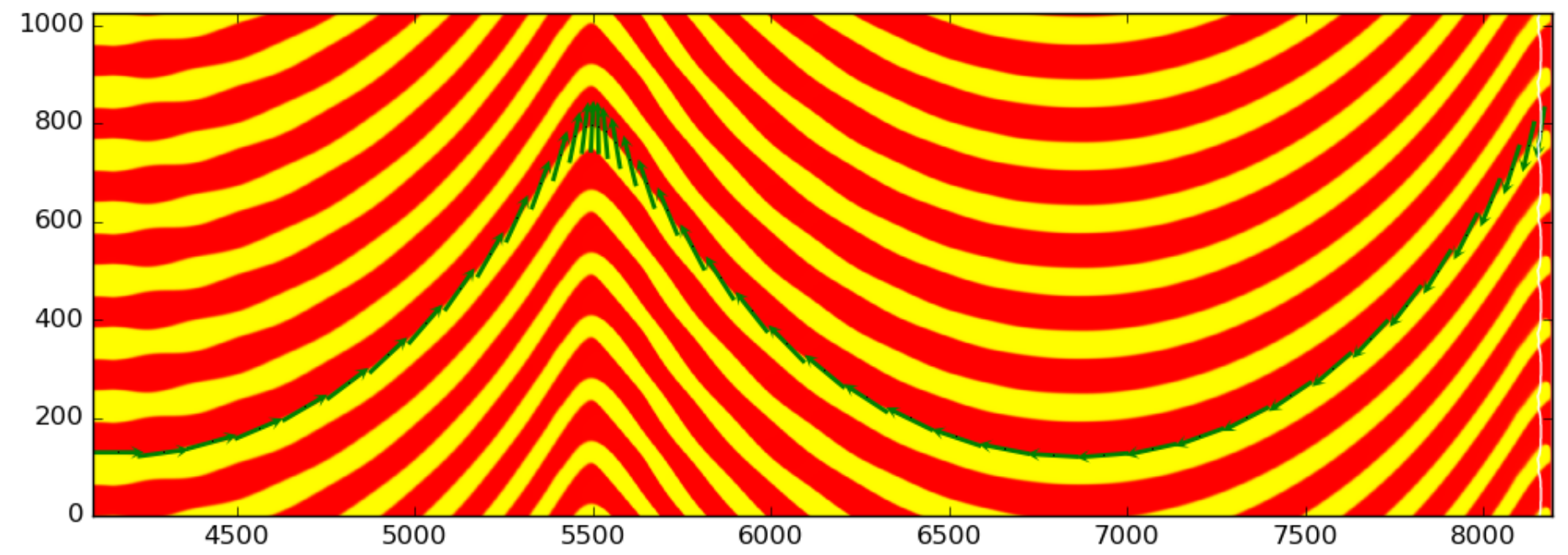


Fig. 8

(a)

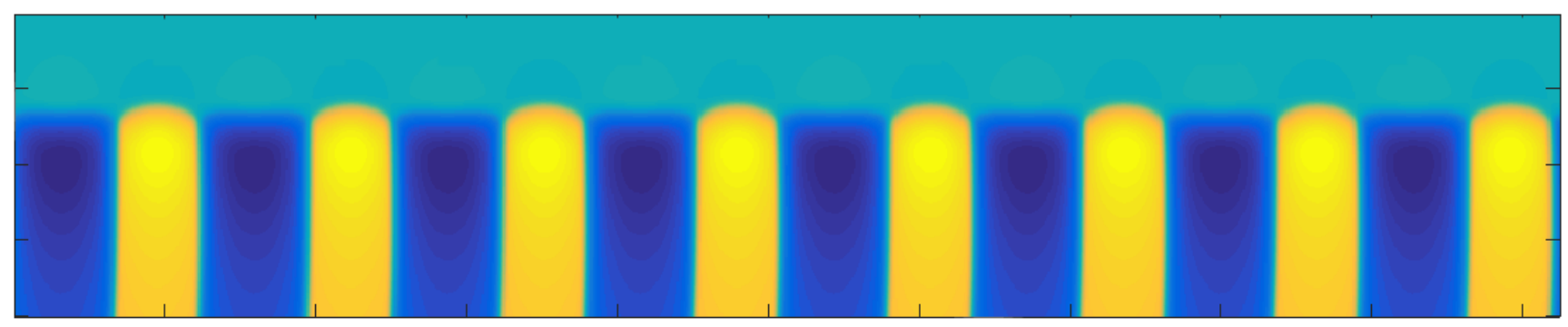

(b)

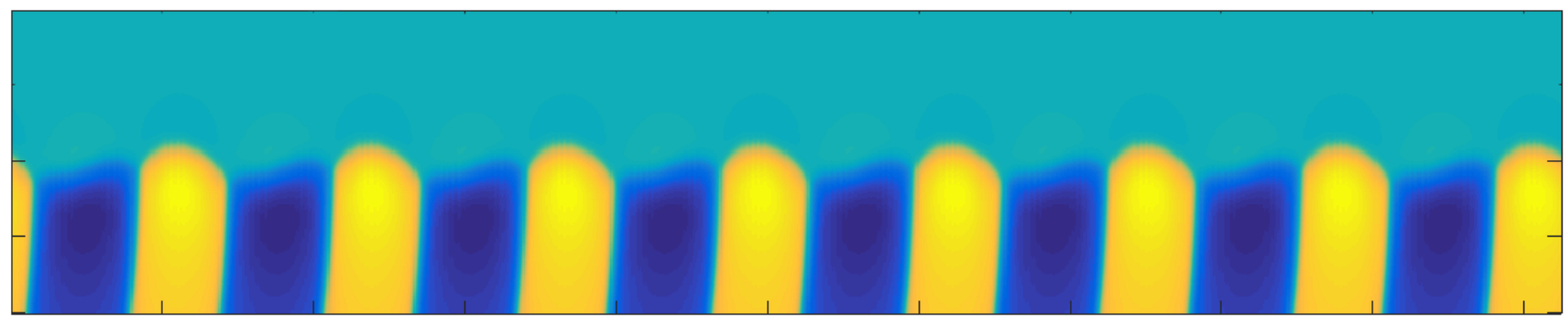


Fig. 9
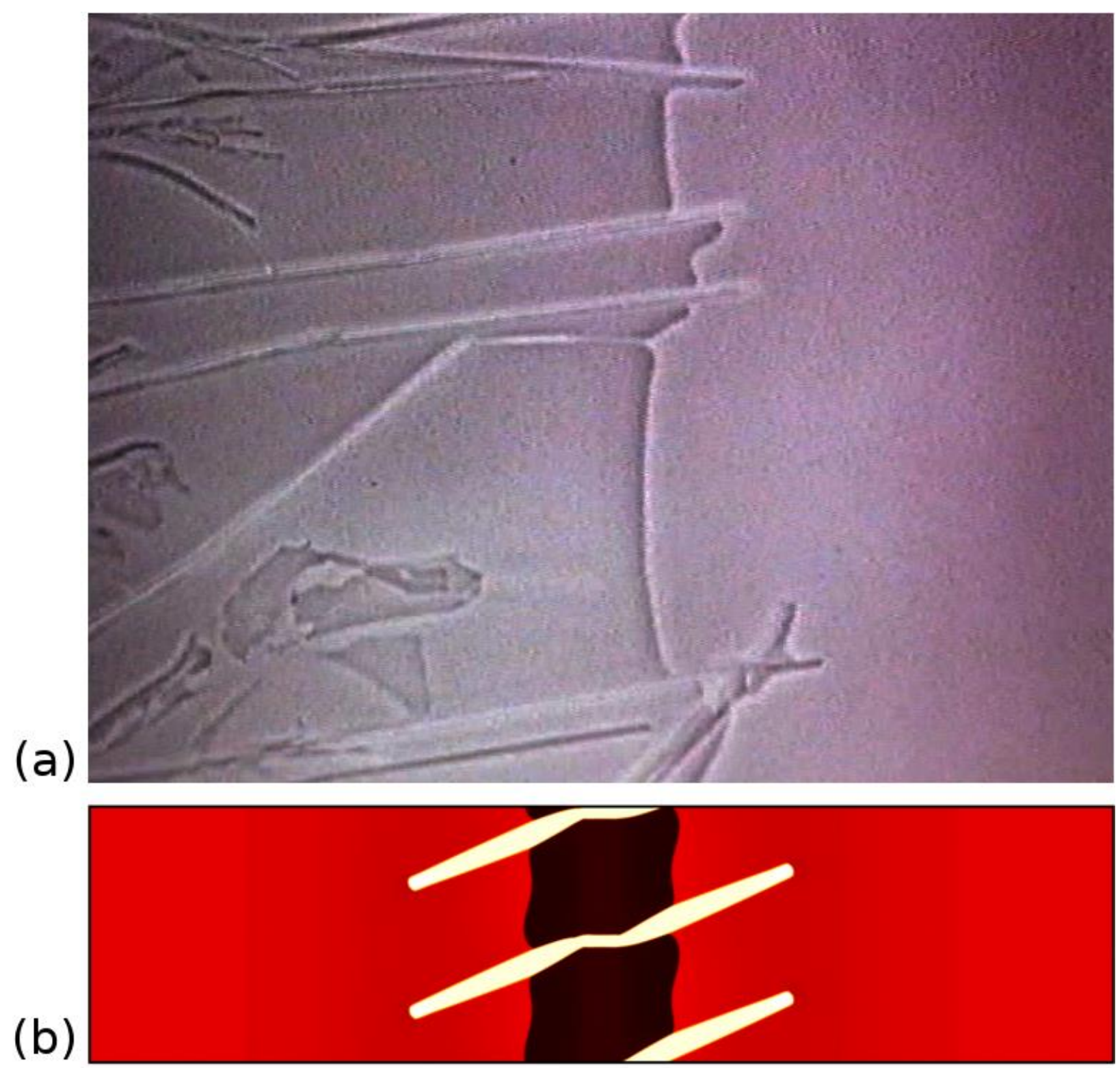
Fig. 10
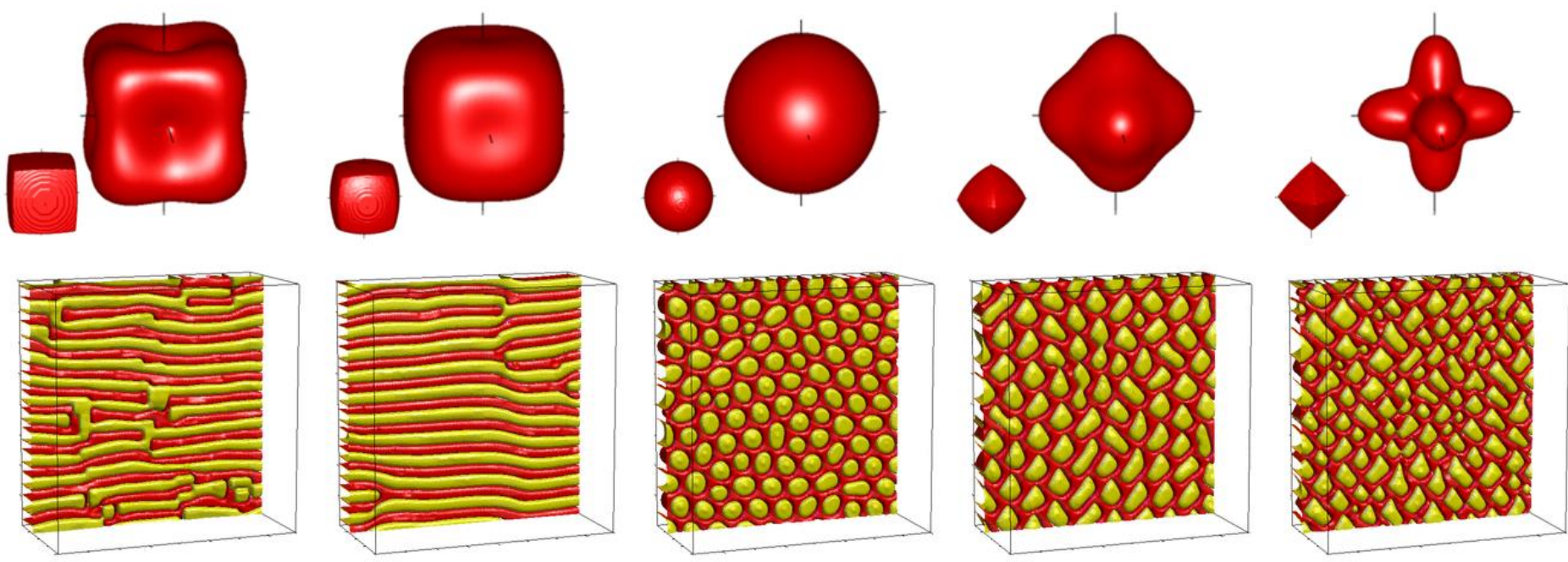
Fig. 11
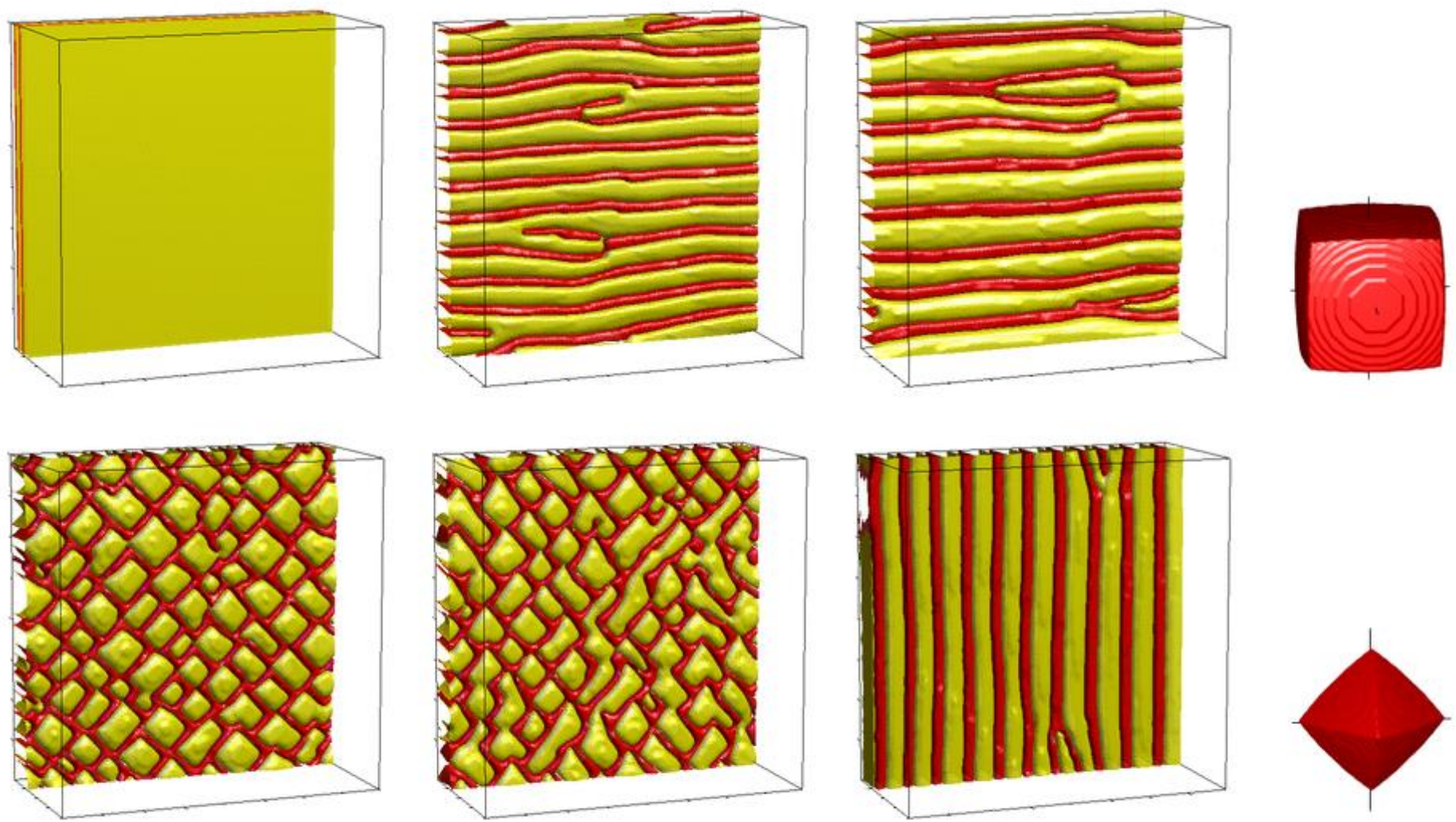
Fig. 12
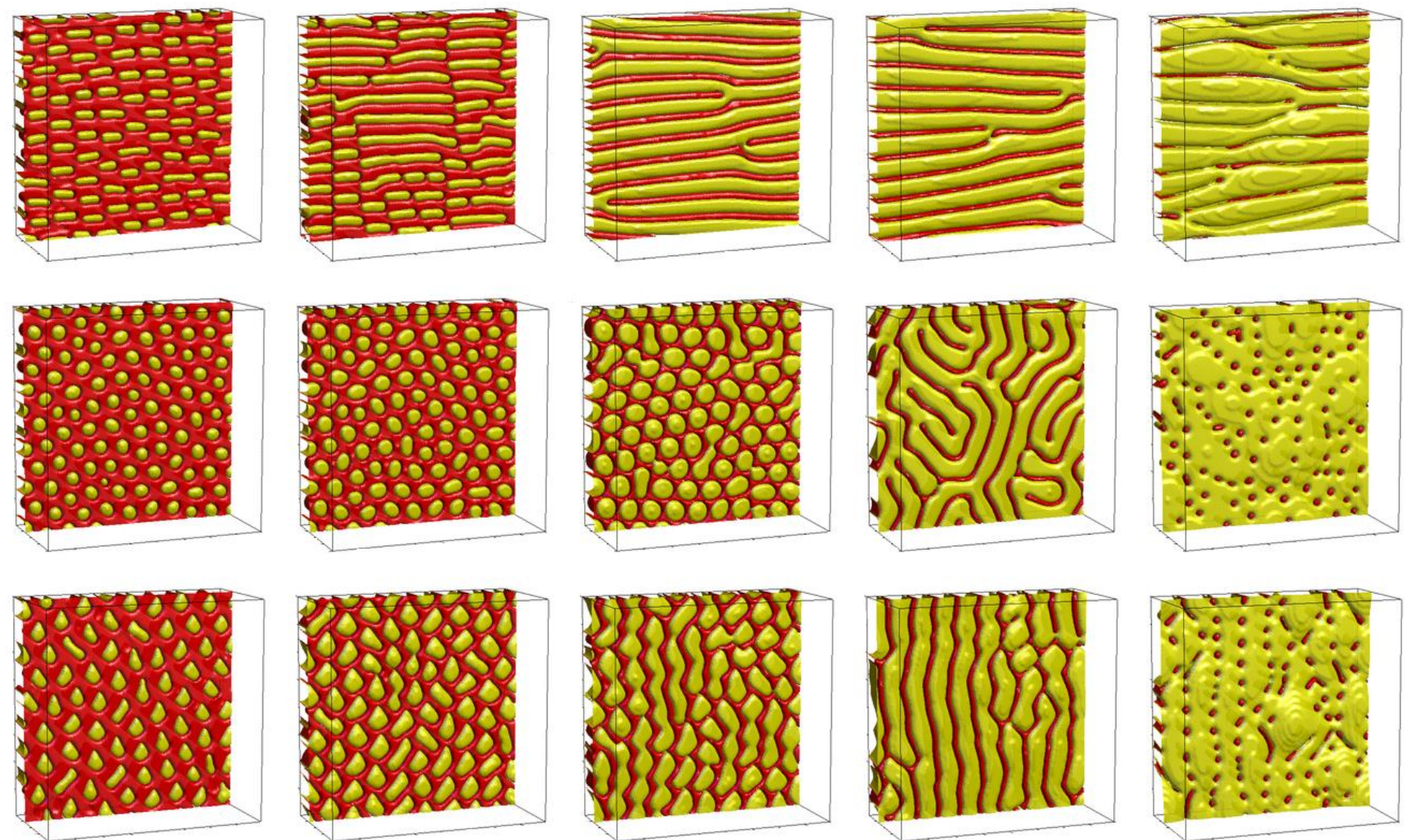
Fig. 13
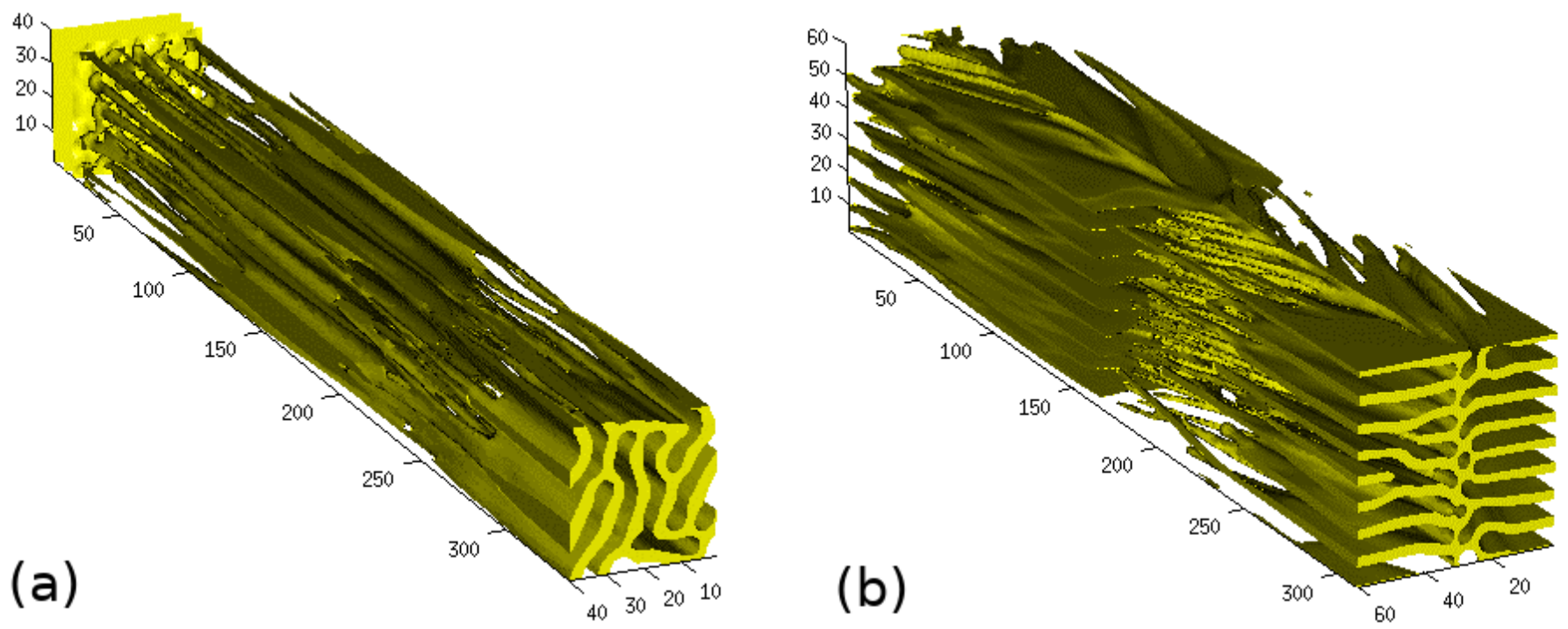
Fig. 14
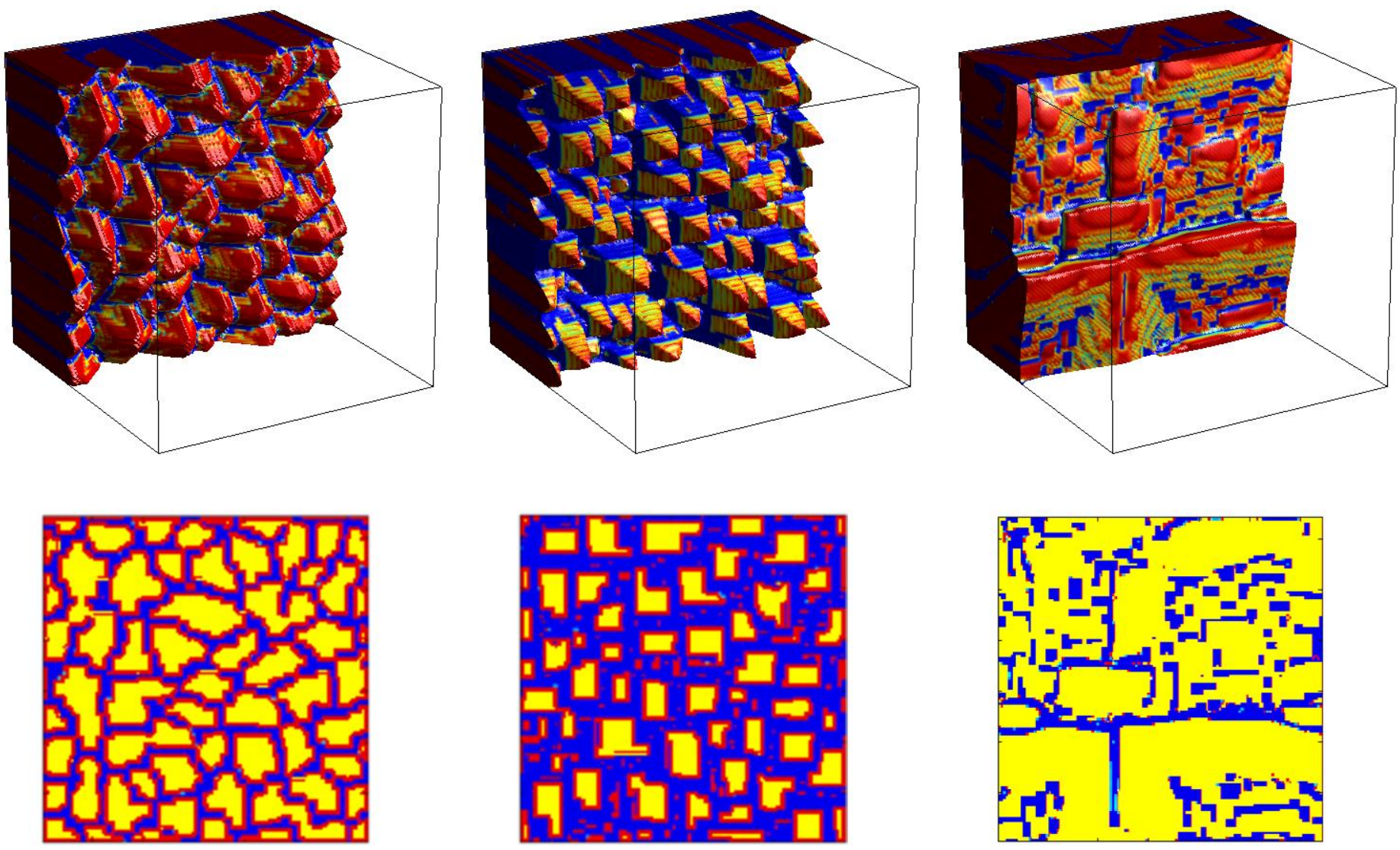
Fig. 15

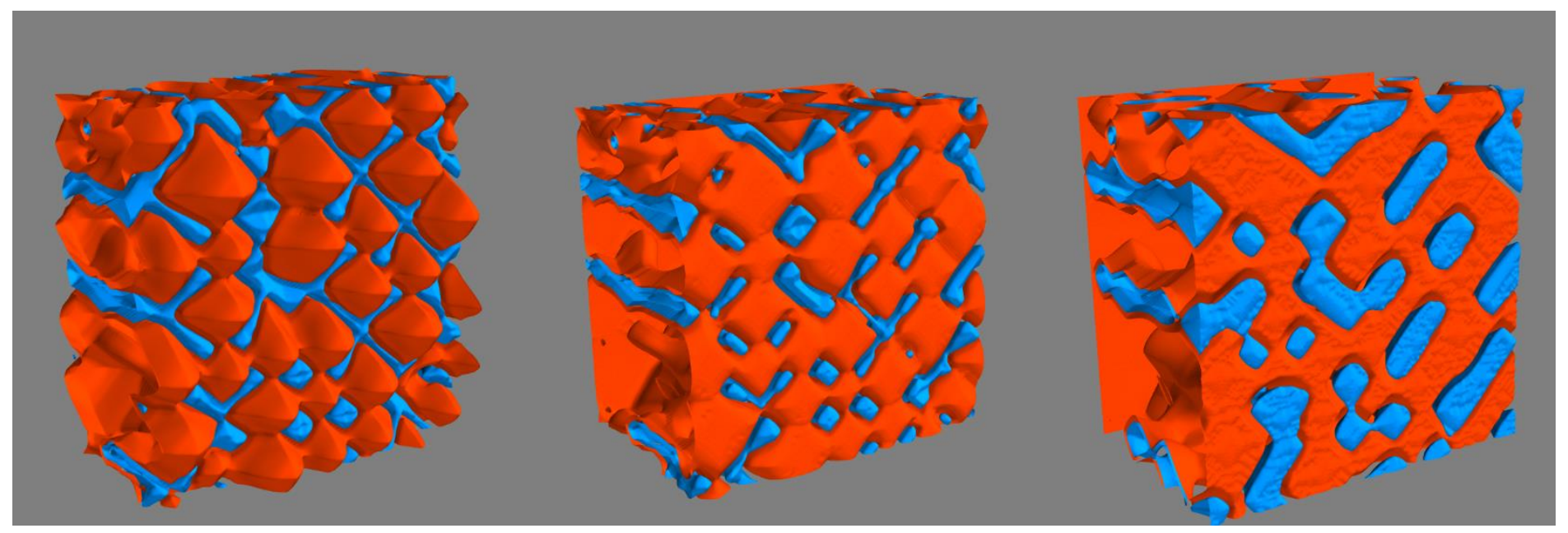


Fig. 16
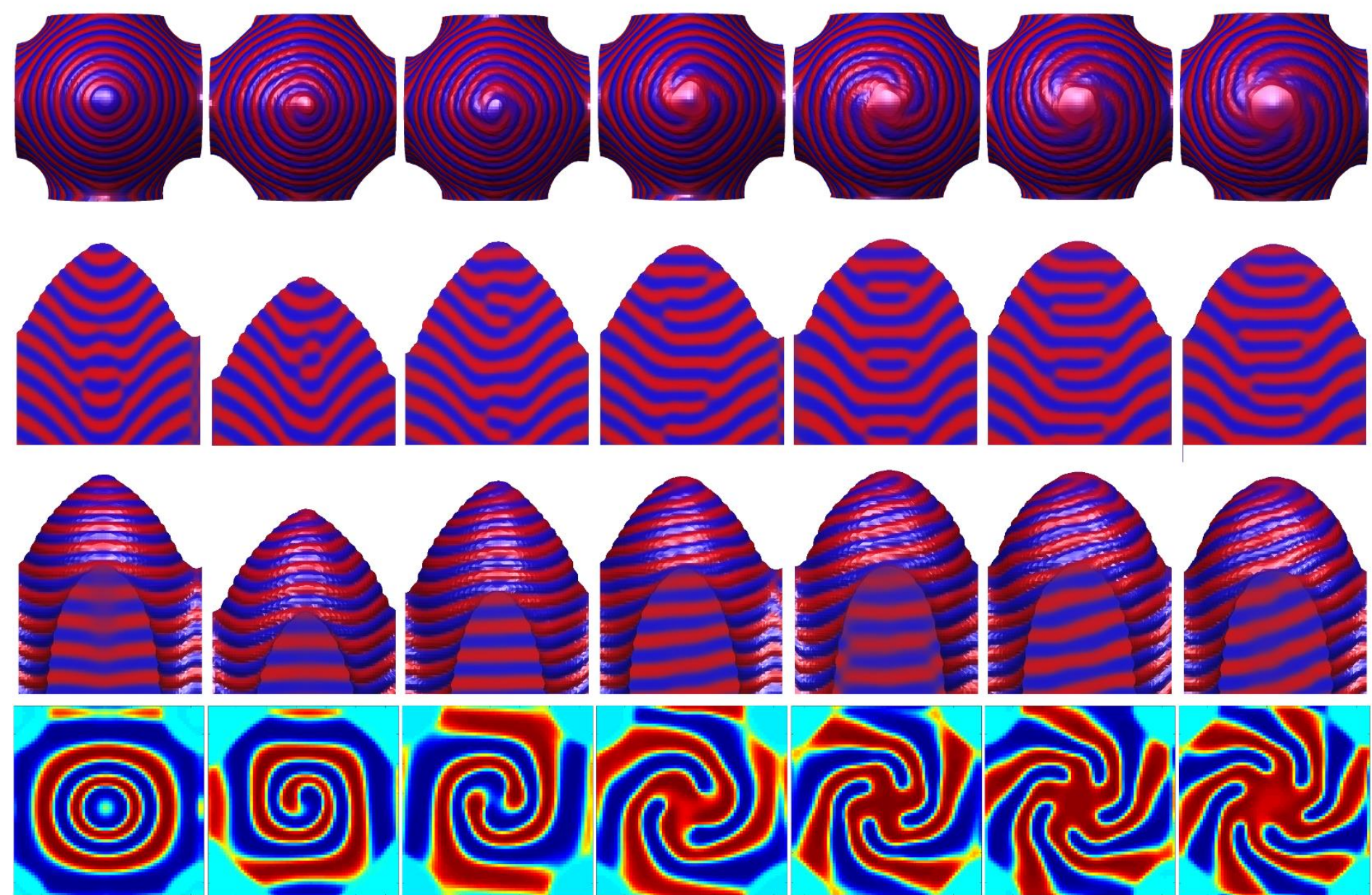